\title{
Diversity and Distribution of Mites (Acari: Ixodida, Mesostigmata, Trombidiformes, Sarcoptiformes) in the Svalbard Archipelago
}

\author{
Anna Seniczak ${ }^{1, *}$, Stanisław Seniczak ${ }^{2}$, Marla D. Schwarzfeld ${ }^{3}$, Stephen J. Coulson ${ }^{4,5}$ and \\ Dariusz J. Gwiazdowicz ${ }^{6}$ \\ 1 Department of Natural History, University Museum of Bergen, University of Bergen, Postboks 7800, \\ 5020 Bergen, Norway \\ 2 Department Evolutionary Biology, Faculty of Biological Sciences, Kazimierz Wielki University, J.K. \\ Chodkiewicza 30, 85-064 Bydgoszcz, Poland; stseni@ukw.edu.pl \\ 3 Canadian National Collection of Insects, Arachnids and Nematodes, Agriculture and Agri-food Canada, \\ 960 Carling Avenue, Ottawa, ON K1A 0C6, Canada; marla.schwarzfeld@canada.ca \\ 4 Swedish Species Information Centre, Swedish University of Agricultural Sciences, SLU Artdatabanken, \\ Box 7007, 75007 Uppsala, Sweden; stephen.coulson@slu.se \\ 5 Department of Arctic Biology, University Centre in Svalbard, P.O. Box 156, 9171 Longyearbyen, Norway \\ 6 Faculty of Forestry, Poznań University of Life Sciences, Wojska Polskiego 71c, 60-625 Poznań, Poland; \\ dariusz.gwiazdoswicz@up.poznan.pl \\ * Correspondence: Anna.Seniczak@uib.no
}

Received: 21 July 2020; Accepted: 19 August 2020; Published: 25 August 2020

\begin{abstract}
Svalbard is a singular region to study biodiversity. Located at a high latitude and geographically isolated, the archipelago possesses widely varying environmental conditions and unique flora and fauna communities. It is also here where particularly rapid environmental changes are occurring, having amongst the fastest increases in mean air temperature in the Arctic. One of the most common and species-rich invertebrate groups in Svalbard is the mites (Acari). We here describe the characteristics of the Svalbard acarofauna, and, as a baseline, an updated inventory of 178 species (one Ixodida, 36 Mesostigmata, 43 Trombidiformes, and 98 Sarcoptiformes) along with their occurrences. In contrast to the Trombidiformes and Sarcoptiformes, which are dominated in Svalbard by species with wide geographical distributions, the Mesostigmata include many Arctic species (39\%); it would thus be an interesting future study to determine if mesostigmatid communities are more affected by global warming then other mite groups. A large number of new species (42 spp.) have been described from Svalbard, including 15 that have so far been found exclusively there. It is yet uncertain if any of these latter species are endemic: six are recent findings, the others are old records and, in most cases, impossible to verify. That the Arctic is still insufficiently sampled also limits conclusions concerning endemicity.
\end{abstract}

Keywords: checklist; Astigmata; Endeostigmata; Oribatida; Prostigmata; climate change; Arctic; Svalbard

\section{Introduction}

The invertebrate fauna of the Svalbard archipelago is amongst the best known for any region in the Arctic [1]. However, the inventory has been collated from studies over a period of 150 years and has never been subjected to a critical review and taxonomic revision. Without an improved understanding of the current fauna it is not possible to understand the current terrestrial ecosystem or determine its response to on-going environmental change. 
The dramatic environmental changes that we see today may be most discernable in polar regions, which possess both a unique flora and fauna and are undergoing the greatest rates of climate change. A particularly interesting area to study this is the Svalbard archipelago that is located at $78^{\circ} \mathrm{N}$, isolated, and with a distinctive and diverse flora and fauna that are poorly studied [2]. Svalbard is currently experiencing one of the fastest temperature increases and one of the highest rates of sea ice loss in the Arctic [3]. During the past 50 years, the mean air temperature in Svalbard has increased by $4{ }^{\circ} \mathrm{C}$, the winter mean air temperature has increased by $7{ }^{\circ} \mathrm{C}$, the snow season duration has decreased by 20 days, and precipitation has increased by $65 \%$ [4]. For example, at Svalbard airport in 2018, the mean annual temperature was $-1.8{ }^{\circ} \mathrm{C}$, winter temperature was $-5.3^{\circ} \mathrm{C}$, and annual precipitation was $252.5 \mathrm{~mm}$ [5]. These factors, as well as others that are tightly connected to the climate (e.g., faster melting of glaciers, changes in permafrost, landslides, avalanches, and flooding), affect the ecosystems in different manners. Even though a few species benefit from a warming climate, for example vertebrates, most Arctic species in Svalbard are experiencing negative effects in the warming environment [6].

Just south of Svalbard, two sea currents meet, a northern branch of the warm North Atlantic Drift and the southerly flowing cold Arctic Current, with consequences for the local climates on the east and west coasts of the archipelago [7] and potentially for species immigration histories, thus affecting species communities. A good example is the distinctive acarofauna of Edgeøya in the east of the archipelago compared to that observed on the west coast [8] and which includes an oribatid species new to science and not so far recorded elsewhere [9].

As a consequence of the increasing temperatures and changes in precipitation patterns, many glaciers are receding and revealing new land surfaces for primary succession. Therefore, regions such as Svalbard offer a unique opportunity to identify the pioneer species that first colonize barren post-glacial habitats and to analyze long-term processes of change, e.g., in invertebrate community assembly [10].

The diversity and richness of the environment is much more than simply lists of species occurring in a given area; it is also the regional specificity of these species, for example, those species that are considered to be typically High Arctic. Changing climatic conditions that determine and stimulate changes in habitats also affect the ranges of some mite species. Several studies have considered changes in mite communities in Svalbard in space and time; their dispersal abilities [11-17], as well as their emergence in new areas or retreat from previously occupied locations [10,18-20]. The uniqueness of polar areas, with their characteristic climates and isolated island distribution that often restricts dispersal processes, limits the range of some species. In this context, knowledge of zoogeography (ranges of occurrence of species) is extremely valuable because this suggests not only the migration routes, but also the ability of selected species to colonize new areas. Nonetheless, the climate of Svalbard is changing fast. These changes will undoubtedly provide opportunities for new species to colonise the region with difficult to foresee outcomes. The consequences of human introductions of invasive and alien species (IAS) have been described in the Antarctic [21] and there are examples of similar introductions in Svalbard [18].

Understanding mite ecology is also important since mites are excellent bioindicators of environmental changes and their presence, or changes in their communities, can be used in biological monitoring of naturally occurring processes as well as the consequences of human impacts, for example, those related to industrial activities in Svalbard's natural environment [20,22].

We here present a review of the mite fauna of Svalbard. These results can be the basis for further analyzes of the acarofauna (e.g., zoogeographic, ecological, taxonomic, and parasitological).

\section{Material and Methods}

The data were extracted from 104 papers published between 1871 and 2020. The localities presented in Figure 1 are based on Table 1. Distribution of Ixodes uriae White, 1852, the only representative of the order Ixodida in Svalbard, follows [23]. The nomenclature and arrangement of the Mesostigmata 
families follows [24,25], while the species nomenclature follows [26] with a few modifications [27,28]. Distribution of the families follows [27-30].

\section{Svalbard}

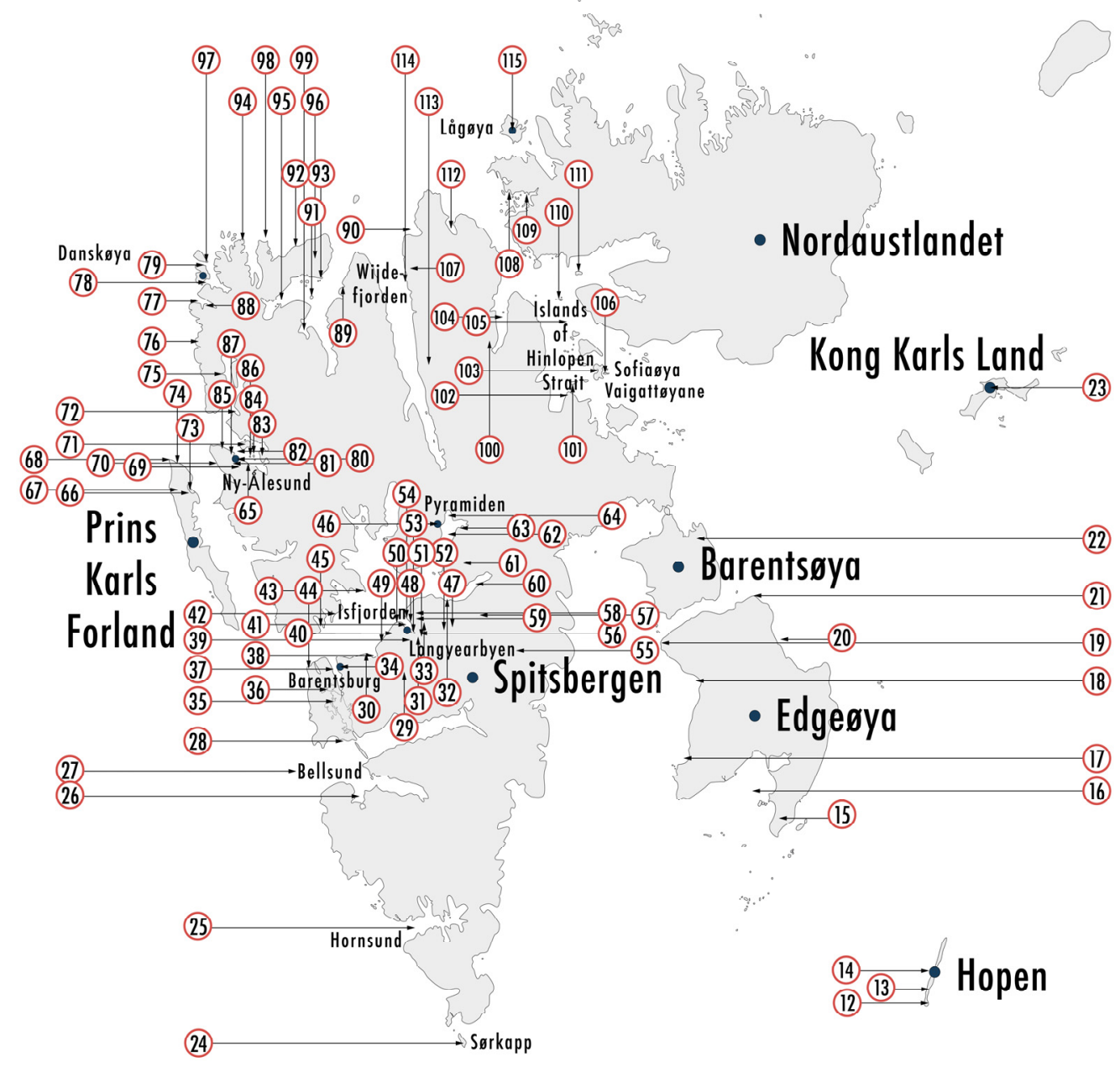

$150 \mathrm{~km}$

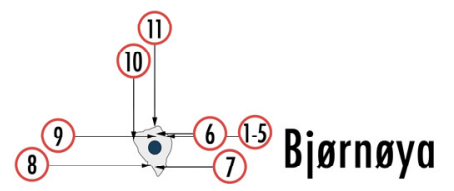

Figure 1. Cont. 


\section{Map Key}

(54) Advent City
(51) Adventdalen
(41) Adventfjorden
(36) Aldegondabreen
(7) Alfredfjellet
(47) Arctowskifjellet
(5) Austervåg
(34) Barentsburg
(22) Barentsøya
(27) Bellsund
(62) Billefjorden
(11) Birds colony
(98) Biskayerhuken
(40) Biørndalen
(20) Blåbukta
(71) Blomstrandhalvøya
(99) Bockfjorden
(43) Bohemanneset
(63) Brucebyen

(15) Negerdalen

(76) Nissenfjella

(29) Nordenskiöld Land

(101) Nordenskiöldøya

(80) Ny-Ålesund

(100) Ny-Friesland

(84) Observasionsholmen

(83) Ossian Sarsfjellet

(64) Petuniabukta

(46) Pyramiden

(26) Recherchefjorden

(96) Reinsdyrflya

(73) Richardlaguna

(113) Ringhorndalen

(17) Russebukta

(57) Sassendalen

(32) Sassenfjorden

(15) Signehamna

(66) Silene Hill

(97) Smeerenburg
(74) Carmichaelpynten

(38) Coleshukta

(114) Dirksbukta

(18) Diskobukta

(8) Ellasjøen

(39) Endulen

(1) Engelskelva

(31) Engelskhytta

(102) Eremitten

(56) Fivelflyene

(72) Fjortende Julibukto

(94) Flathuken

(109) Florabukta

(110) Fosterøyane

(68) Fuglehuken fyr

(2) Fugleodden

(61) Gipsdalen

(35) Gronfjordbreen

(37) Grønfjorden
(88) Großer Trichter

(49) Grumantbyen

(iii) Gyldénøyane

(58) Hanaskogdalen

(6) Hellevatnet

(52) Helvetiadalen

(105) Hinlopenstretet

(59) Hiorthfjellet

(48) Hiorthhamn

(14) Hopen

(13) Hopen radio

(25) Hornsund

(53) Isdammen

(44) Isfiord radio

(42) Isfjorden

(21) Kapp Heuglin

(30) Kapp Laila

(19) Kapp Lee

(70) Kiærstranda
(108) Kinnvika

(87) Knudsenheia

(12) Koefoedodden

(81) Kolhaugen

(23) Kong Karls Land

(82) Kongsfjorden

(65) Krykkjefjellet

(115) Lågøya

(107) Lakssiøen

(95) Liefdefjorden

(104) Lomfjorden

(55) Longyearbyen

(67) MacKenziedale

(77) Magdalenefiorden

(33) Mälardalen

(69) Midtre Lovénbreen

(4) Mosevainet

(90) Mosselbukta

(89) Mushamna
(106) Sofiaøya
(78) Sorgattet
(112) Sorgfjorden
(24) Sorkapp
(91) Station Islands at Liefdefiorden
(9) Stevatnet
(86) Storholmen
(85) Stuphallet
(92) Sven Olssonodden
(10) Teltvika
(60) Tempelfjorden
(16) Tjuvfjorden
(45) Trygghamna
(3) Tunheim
(28) Vårsolbukta
(50) Vestpynten
(19) Virgohamna
(103) Von Otterøya
(93) Worsleyneset

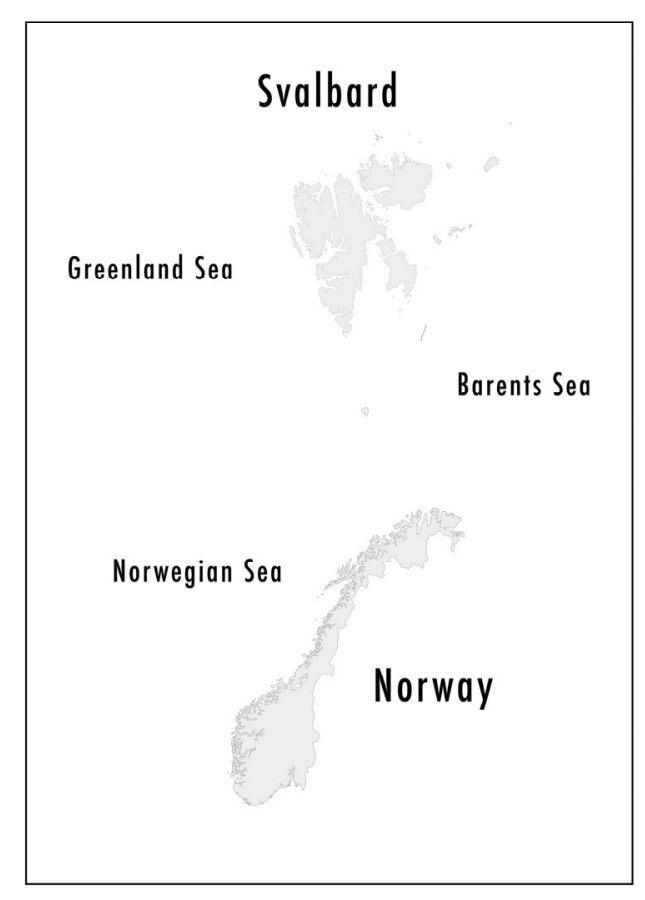

Figure 1. Localities in Svalbard where mites were found.

The arrangement of the Trombidiformes families follow [31] with the exception of the Eriophyidae that is included in the Endeostigmata, as suggested by [32,33]. Names and distributions of the Bdellidae follow [34]; Cunaxidae- [35]; Cocceupodidae and Eupodidae- [36,37] for nomenclature, and [38,39] for distributions; Penthaleidae-[40]; Penthalodidae-[41]; Rhagidiidae-[42-44]; Halacaridae-[45]; Triophtydeiidae — [46] for taxonomy, and [47,48] for distribution; Tydeidae- $[49,50]$; Iolinidae $-[51,52]$ 
for taxonomy, and [47,53] for distributions; Sperchontidae-[48,54,55]; Trombidiidae-[56]; Syringophilidae-[57]; Stigmaeidae — [58]; Tetranychidae-[59,60]; and Pygmephoridae-[61].

The nomenclature and arrangement of the Sarcoptiformes families follow [62,63]. For the Endeostigmata, the nomenclature and arrangement of families follow [63,64]. The distribution of Nanorchestidae follow [65]; Alicorhagiidae-[66]; and Eriophyidae-[67]. The species names of the Oribatida (without Astigmata) follow [68] with a few exceptions [69,70] and their distribution is given after $[9,68,71,72]$. The Astigmata species (families Acaridae, Alloptidae, and Avenzoariidae) follow $[73,74]$ and their distribution is according to [75].

Full names of species are provided in Table 1, while in other tables and figures abbreviations are used. The species within families are organized alphabetically. The names of localities follow in alphabetical order (Table 1).

\section{Results}

\subsection{Ixodida}

Only one species, the seabird tick (Ixodes uriae), is known from Svalbard. This species has a wide geographical distribution (Table 1) but in Svalbard has been found only recently on Bjørnøya and Spitsbergen [76-78].

\subsection{Mesostigmata}

Thus far, 36 species of Mesostigmata from 13 families have been found in Svalbard (Table 1). Amongst these families, the richest in species is the Ascidae (12 spp.), followed by the Zerconidae (6 spp.); these two families contribute almost $50 \%$ of mesostigmatic alpha diversity in Svalbard (Figure 2).

The peculiarity of the mesostigmatic mite communities of Svalbard compared to other mite groups is manifested by the description of one new genus (Arctoseius) and seven species [Halolaelaps coulsoni Gwiazdowicz \& Teodorowicz, 2017; H. gerlachi Hirschmann, 1966; Antennoseius (Vitzthumia) oudemansi (Thor, 1930); Arctoseius laterincisus Thor, 1930; Proctolaelaps parvanalis (Thor, 1930); Neoseiulus grumantensis Kolodochka \& Gwiazdowicz, 2014; and N. magnanalis (Thor, 1930)], all first described from the largest island of the archipelago-Spitsbergen. Moreover, based on recently collected samples from this island, redescriptions of several species have been published: Zercon solenites Haarløv, 1942; Antennoseius (Vitzthumia) oudemansi; Arctoseius haarlovi Lindquist, 1963; Proctolaelaps parvanalis; Neoseiulus ellesmerei (Chant \& Hansell, 1971); and N. magnanalis [27-29,79,80]. 
Table 1. List of mites of the Svalbard archipelago.

\begin{tabular}{|c|c|c|c|}
\hline No. & Taxon & Distribution & Localities \\
\hline & IXODIDA & & \\
\hline \multirow{3}{*}{1.} & Ixodidae & & \\
\hline & Ixodes uriae White, 1852 & $\begin{array}{c}\text { Afrotropical, Australasian, Neotropical, } \\
\text { Holarctic }\end{array}$ & Bjørnøya [77]; Spitsbergen: Ossian Sarsfjellet [76-78] \\
\hline & MESOSTIGMATA & & \\
\hline \multirow[t]{2}{*}{2} & $\begin{array}{c}\text { Dinychidae } \\
\text { Uroseius acuminatus (C.L. Koch, 1847) }\end{array}$ & Palearctic & Spitsbergen $[81,82]$ \\
\hline & Zerconidae & & \\
\hline 3. & Zercon andrei Sellnick, 1958 & Palearctic & $\begin{array}{c}\text { Bjørnøya: Alfredfjellet, “Birds colony", Teltvika [29,30,83]; Spitsbergen: Hornsund } \\
\text { [84] }\end{array}$ \\
\hline 4. & Z. curiosus Trägårdh, 1910 & Palearctic & Spitsbergen: Longyearbyen [85] \\
\hline 5. & Z. forsslundi Sellnick, 1958 & Palearctic & $\begin{array}{c}\text { Edgeøya: Diskobukta, Kapp Heuglin, Kapp Lee, Negerdalen, Russebukta [8]; } \\
\text { Hopen: Koefoedodden, Norwegian Meteorological Institute Station [86]; } \\
\text { Nordaustlandet: Kinnvika [30,87]; Spitsbergen: Adventdalen, Billefjord, } \\
\text { Bjørndalen, Endalen, Hornsund, Ny-Ålesund, Ossian Sarsfjellet, Petuniabukta, } \\
\text { Storholmen, Trygghamna [10,20,30,85,88-90] }\end{array}$ \\
\hline 6. & Z. solenites Haarløw, 1942 ** & Arctic & $\begin{array}{c}\text { Edgeøya: Diskobukta, Kapp Lee [8]; Hopen: Koefoedodden, Norwegian } \\
\text { Meteorological Institute Station [86]; Nordaustlandet: Kinnvika [29,87]; } \\
\text { Spitsbergen: Adventdalen, Bjørndalen, Blomstrandhalvøya, Endalen, Fjortende } \\
\text { Julibukta, Grønfjorden, Magdalenefjorden, Petuniabukta, Storholmen, } \\
\text { Vårsolbukta [20,27,30,89,90] }\end{array}$ \\
\hline 7. & Z. triangularis C.L. Koch, 1836 & Palearctic & Spitsbergen: Adventdalen, Barentsburg, Hanaskogdalen, Hjorthhammn [91] \\
\hline 8. & Z. zelawaiensis Sellnick, 1944 & Palearctic & Bjørnøya: Alfredfjellet [88]; Spitsbergen: Hornsund [83] \\
\hline 9. & $\begin{array}{c}\text { Parasitidae } \\
\text { Paragamasus (Aclerogamasus) insertus } \\
\text { (Micherdzinski, 1969) }\end{array}$ & Palearctic & Spitsbergen: Barentsburg $[18,19]$ \\
\hline 10. & Vulgarogamasus immanis (Berlese, 1904) & Palearctic & Spitsbergen: Barentsburg [92] \\
\hline 11. & V. remberti (Oudemans 1912) & Palearctic & Spitsbergen: Barentsburg $[18,19]$ \\
\hline 12. & $\begin{array}{c}\text { Digamasellidae } \\
\text { Dendrolaelaps foveolatus (Leitner, 1949) }\end{array}$ & Palearctic & Spitsbergen: Pyramiden [20] \\
\hline 13. & $\begin{array}{c}\text { Halolaelapidae } \\
\text { Halolaelaps coulsoni Gwiazdowicz \& } \\
\text { Teodorowicz, } 2017^{*,+}\end{array}$ & Arctic & Spitsbergen: Pyramiden [93] \\
\hline 14. & H. gerlachi Hirschmann, $1966^{*,+}$ & Arctic & Spitsbergen: Ny-Ålesund [94] \\
\hline 15. & Saprosecans baloghi Karg, 1964 & Palearctic & Edgeøya: Diskobukta [8] \\
\hline
\end{tabular}


Table 1. Cont

\begin{tabular}{|c|c|c|c|}
\hline No. & Taxon & Distribution & Localities \\
\hline 16. & $\begin{array}{c}\text { Ologamasidae } \\
\text { Gamasellus borealis (C.L. Koch, 1879) }\end{array}$ & Palearctic & Spitsbergen: Dirksbukta [7] \\
\hline 17. & $\begin{array}{c}\text { Eviphididae } \\
\text { Thinoseius spinosus (Willmann, 1939) }\end{array}$ & Palearctic & Spitsbergen: Longyearbyen, Storholmen $[90,95]$ \\
\hline \multirow[t]{2}{*}{18.} & $\begin{array}{c}\text { Macrochelidae } \\
\text { Macrocheles muscaedomesticae (Scopoli, } \\
1772 \text { ) }\end{array}$ & Palearctic & Spitsbergen: Barentsburg [84] \\
\hline & & \multicolumn{2}{|c|}{ Ascidae } \\
\hline 19. & $\begin{array}{l}\text { Antennoseius (Vitzthumia) oudemansi } \\
\quad\left(\text { Thor, 1930) }{ }^{* * * *}\right.\end{array}$ & Arctic & $\begin{array}{c}\text { Bjørnøya: “Birds colony" [83]; Edgeøya: Diskobukta, Kapp Lee, } \\
\text { Russebukta [8]; Hopen: Koefoedodden, Norwegian } \\
\text { Meteorological Institute Station [86]; Nordaustlandet: Kinnvika } \\
\text { [30,79,87]; Spitsbergen: Adventdalen, Barentsburg, Bjørndalen, } \\
\text { Endalen, Florabukta, Hiorthhamn, Magdalenefjorden, } \\
\text { Petuniabukta, Pyramiden, Storholmen, Vestpynten } \\
\text { [19,20,22,30,79,85,88-91] }\end{array}$ \\
\hline 20. & Arctoseius babenkoi Makarova, 1999 & Palearctic & Spitsbergen: Hornsund, Pyramiden $[20,84,96]$ \\
\hline 21. & A. cetratus (Sellnick, 1940) & Holarctic & $\begin{array}{c}\text { Spitsbergen [96] } \\
\text { Biørngya. "Birds colony" [83] · Fdogya. Diskobukta Kann Leo }\end{array}$ \\
\hline 22. & A. haarlovi Lindquist, 1963 ** & Arctic & $\begin{array}{c}\text { Bjørnøya: “Birds colony” [83]; Edgeøya: Diskobukta, Kapp Lee, } \\
\text { Negerdalen, Russebukta [8]; Hopen: Koefoedodden, } \\
\text { Norwegian Meteorological Institute Station [86]; Spitsbergen: } \\
\text { Adventdalen, Barentsburg, Bjørndalen, Endalen, Hornsund, } \\
\text { Longyearbyen, Magdalenefjorden, Mushamna, Ny-Ålesund, } \\
\text { Petuniabukta, Pyramiden, Storholmen, Vestpynten } \\
\text { [10,19,20,22,30,80,89,90,97] }\end{array}$ \\
\hline 23. & A. laterincisus Thor, $1930^{*,+}$ & Arctic & $\begin{array}{c}\text { Spitsbergen: Adventdalen, Barentsburg, Hanaskogdalen, } \\
\text { Hjorthhamn [91] }\end{array}$ \\
\hline 24. & A. multidentatus Evans, 1955 & Arctic & $\begin{array}{l}\text { Edgeøya: Blåbukta, Diskobukta, Kapp Heuglin, Kapp Lee, } \\
\text { Negerdalen, Russebukta [8]; Hopen: Koefoedodden, } \\
\text { Norwegian Meteorological Institute Station [86]; } \\
\text { Nordaustlandet: Florabukta, Kinnvika [30,87]; Spitsbergen: } \\
\text { Adventdalen, Barentsburg, Billefjorden, Endalen, Grønfjorden, } \\
\text { Hornsund, Magdalenefjorden, Mushamna, Ny-Ålesund, } \\
\text { Petuniabukta, Pyramiden, Storholmen, Sørkapp, Vårsolbukta } \\
\text { [10,19,20,30,88-90,96] }\end{array}$ \\
\hline 25. & A. ornatus Lindquist, 1961 & Holarctic & Spitsbergen [96] \\
\hline
\end{tabular}


Table 1. Cont

\begin{tabular}{|c|c|c|c|}
\hline No. & Taxon & Distribution & Localities \\
\hline 26. & A. tajmyricus Petrova \& Makarova, 1991 & Palearctic & $\begin{array}{l}\text { Bjørnøya: Alfredfjellet [83]; Spitsbergen: Hornsund [88,96] } \\
\text { Edgeøya: Blåbukta, Kapp Heuglin, Negerdalen [8]; }\end{array}$ \\
\hline 27. & A. tschernovi Makarova, 2000 & Arctic & $\begin{array}{l}\text { Nordaustlandet: Kinnvika [30,87]; Spitsbergen: Adventdalen, } \\
\text { Endalen, Hornsund }[30,89]\end{array}$ \\
\hline 28. & A. weberi Evans, 1955 & Arctic & $\begin{array}{l}\text { Bjørnøya: Alfredfjellet, „,Birds colony” [83]; Edgeøya: Kapp } \\
\text { Heuglin, Kapp Lee, Negerdalen, Russebukta [8]; Spitsbergen: } \\
\text { Adventdalen, Bjørndalen, Endalen, Ossian Sarsfjellet, } \\
\text { Petuniabukta, Vestpynten, Vårsolbukta }[20,22,30,88,89,96]\end{array}$ \\
\hline 29. & $\begin{array}{l}\text { Zerconopsis labradorensis Evans \& Till, } \\
\qquad 1960\end{array}$ & Arctic & Bjørnøya: Alfredfjellet, “Birds colony” [83]; Spitsbergen [84] \\
\hline 30. & Z. muestairi (Schweizer, 1949) & Palearctic & Bjørnøya: Teltvika [29,30] \\
\hline 31. & Proctolaelaps parvanalis (Thor, 1930$)^{*, * *}$ & Arctic & $\begin{array}{c}\text { Bjørnøya: Alfredfjellet, “Birds colony” [83]; Edgeøya: Blåbukta, } \\
\text { Diskobukta, Kapp Lee, Russebukta [8]; Spitsbergen: } \\
\text { Adventdalen, Bjørndalen, Endalen, Hiorthhamn, } \\
\text { Longyearbyen, Mälardalen, Ny-Ålesund, Petuniabukta } \\
\text { [10,20,22,29,30,85,89,91] }\end{array}$ \\
\hline 32. & $\begin{array}{c}\text { Phytoseiidae } \\
\text { Neoseiulus ellesmerei (Chant \& Hansell, } \\
\text { 1971) }\end{array}$ & Arctic & $\begin{array}{c}\text { Edgeøya: Russebukta [8,28]; Spitsbergen: Adventdalen, } \\
\text { Endalen, Longyearbyen [28] }\end{array}$ \\
\hline 33. & $\begin{array}{l}\text { N. grumantensis Kolodochka \& } \\
\text { Gwiazdowicz, } 2014^{*,+}\end{array}$ & Arctic & Spitsbergen: Longyearbyen, Petuniabukta [28] \\
\hline \multirow[t]{2}{*}{34.} & N. magnanalis (Thor, 1930) *,**,+ & Arctic & $\begin{array}{l}\text { Spitsbergen: Adventdalen, Hiorthhamn, Mälardalen, } \\
\text { Ny-Ålesund, Petuniabukta }[10,20,22,28,30,85,89,91]\end{array}$ \\
\hline & Dermanyssidae & & \\
\hline \multirow[t]{2}{*}{35.} & Dermnanyssus hirundinis (Hermann, 1804) & Holarctic & $\begin{array}{c}\text { Edgeøya: Blåbukta [8]; Spitsbergen: Longyearbyen, } \\
\text { Ny-Âlesund [90,98] }\end{array}$ \\
\hline & Laelapidae & & \\
\hline 36. & Haemogamasus ambulans (Thorel, 1872) & Holarctic & $\begin{array}{c}\text { Bjørnøya [99]; Spitsbergen: Adventdalen, Endalen, } \\
\text { Grumantbyen, Fjortende Julibukta, Ossian Sarsfjellet, } \\
\text { Storholmen }[90,91,100]\end{array}$ \\
\hline 37. & Laelaps hilaris C.L. Koch, 1836 & Holarctic & Spitsbergen: Grumantbyen [100] \\
\hline & TROMBIDIFORMES & & \\
\hline & Prostigmata & & \\
\hline
\end{tabular}


Table 1. Cont.

\begin{tabular}{|c|c|c|c|}
\hline No. & Taxon & Distribution & Localities \\
\hline \multicolumn{4}{|c|}{ Bdellidae } \\
\hline 38. & Bdella longicornis (Linnaeus, 1758) & Holarctic, Neotropic, Indomalayan & $\begin{array}{l}\text { Bjørnøya: south [99,101]; Prins Karls Forland: MacKenziedale, } \\
\text { Richardlaguna almost to Carmichaelpynten, Silene Hill } \\
\text { [99,101]; Spitsbergen: Adventfjorden, Bellsund, Billefjorden, } \\
\text { Bohemanneset, Brucebyen, Dirksbukta, Eremitten, Gipsdalen, } \\
\text { Hiorthhamn, Sorgfjorden, Station Islands at Liefdefjorden, } \\
\text { Salmon Lake at Dirksbukta [7,91,99,101,102] }\end{array}$ \\
\hline 39. & B. semiscutata Thor, 1930 * & Palearctic & Spitsbergen: Engelskhytta, Hiorthfjellet, Longyearbyen $[91,103]$ \\
\hline 40. & Odontoscirus lapidaria (Kramer, 1881) & Cosmopolitan & $\begin{array}{l}\text { Bjørnøya [104]; Spitsbergen: Barentsburg [91] } \\
\text { Bjørnøya: Ellasjøen, south [99,101,105]; Prins Karls Forland: } \\
\text { MacKenziedale, Silene Hill [99,101]; Spitsbergen: }\end{array}$ \\
\hline 41. & Cyta latirostris (Hermann, 1804) & Cosmopolitan & $\begin{array}{l}\text { Adventfjorden, Barentsburg, Billefjorden, Brucebyen, } \\
\text { Engelskhytta, “Großer Trichter", Grønfjorden, Longyearbyen, } \\
\text { Magdalenefjorden, Salmon Lake at Dirksbukta, } \\
{[7,91,99,101,103] \text {; Svalbard [106] }}\end{array}$ \\
\hline 42. & Neomolgus capillatus (Kramer, 1881) & Palearctic, Australasian & Spitsbergen: Hiorthhamn [91] \\
\hline 43. & N. littoralis (Linnaeus, 1758) & Holarctic & $\begin{array}{l}\text { Bjørnøya: south [99,101,104,105]; Prins Karls Forland: Silene } \\
\text { Hill [99,101]; Islands of Hinlopenstretet: Fosterøyane, } \\
\text { Gyldénøyane, Vaigattøyane including Von Otterøya and } \\
\text { Nordenskiöldøya [7]; Spitsbergen: Adventfjorden, Billefjorden, } \\
\text { Bohemanneset, Brucebyen, Eremitten, Grønfjorden, between } \\
\text { Hiorthhamn and Advent City, Isfjorden, Liefdefjorden, } \\
\text { Ny-Friesland, Salmon Lake at Dirksbukta, Sassenfjorden, } \\
\text { Sorgfjorden, south side of Reinsdyrflya [7,91,99,101,102,105] }\end{array}$ \\
\hline 44. & N. pallipes (C.L. Koch, 1879) & Holarctic & $\begin{array}{c}\text { Spitsbergen: Adventfjorden, Billefjorden, Brucebyen, south } \\
\text { side of Reinsdyrflya }[7,99,101]\end{array}$ \\
\hline 45. & $\begin{array}{c}\text { Cunaxidae } \\
\text { Cunaxoides croceus (C.L. Koch, 1838) }\end{array}$ & Holarctic & Spitsbergen: “Großer Trichter" [103] \\
\hline \multicolumn{4}{|c|}{ Cocceupodidae } \\
\hline 46. & Cocceupodes mollicellus C.L. Koch 1838 & Holarctic & $\begin{array}{c}\text { Spitsbergen: “Großer Trichter", Hiorthhamn, Longyearbyen, } \\
\text { Magdalenefjorden }[91,103,106]\end{array}$ \\
\hline \multicolumn{4}{|c|}{ Eupodidae } \\
\hline 47. & Eupodes variegatus C.L. Koch, 1838 & Holarctic, Afrotropical & $\begin{array}{l}\text { Bjørnøya: Engelskelva, Fugleodden [91]; Spitsbergen: } \\
\text { Hanaskogdalen, Hiorthhamn, Longyearbyen? }[91,103,106]\end{array}$ \\
\hline 48. & Neoprotereunetes borneri (Thor, 1934) ${ }^{*, 1}$ & Holarctic & $\begin{array}{c}\text { Spitsbergen: “Großer Trichter", Magdalenefjorden, } \\
\text { Tempelfjorden }[66,103]\end{array}$ \\
\hline
\end{tabular}


Table 1. Cont

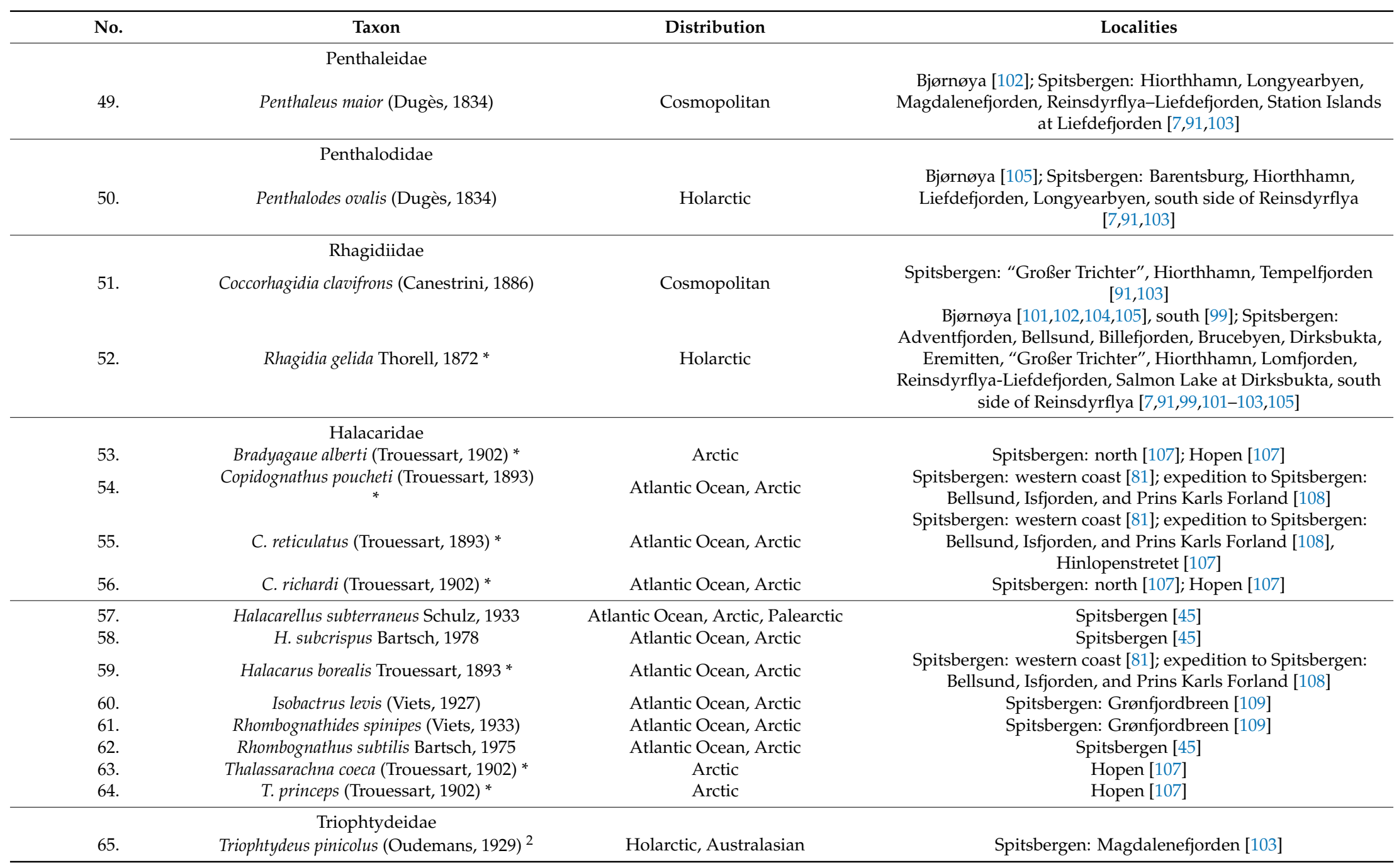


Table 1. Cont

\begin{tabular}{|c|c|c|c|}
\hline No. & Taxon & Distribution & Localities \\
\hline \multirow[b]{2}{*}{66.} & Tydeidae & & \\
\hline & Tydeus langei Thor, $1934^{3, *,+}$ & Arctic & $\begin{array}{c}\text { Spitsbergen: “Großer Trichter”, Magdalenefjorden, } \\
\text { Tempelfjorden [103] }\end{array}$ \\
\hline 67. & T. svalbardensis Thor, $1932^{3, *,+}$ & Arctic & $\begin{array}{l}\text { Bjørnøya: Engelskelva, Fugleodden [91,110]; Spitsbergen: } \\
\text { “Großer Trichter", Hanaskogdalen, Hiorthhamn, } \\
\text { Longyearbyen, Magdalenefjorden, Tempelfjorden }[91,103]\end{array}$ \\
\hline & Iolinidae & & \multirow{4}{*}{$\begin{array}{c}\text { Spitsbergen: “Großer Trichter", Magdalenefjorden, } \\
\text { Tempelfjorden [103]; Svalbard [47] } \\
\text { Spitsbergen: Tempelfjorden [103]; Svalbard [47] } \\
\text { Spitsbergen: “Großer Trichter", Magdalenefjorden [103]; } \\
\text { Svalbard [47] }\end{array}$} \\
\hline 68. & Tydaeolus globifer (Thor, 1931) & Arctic & \\
\hline 69. & T. tenuiclaviger (Thor, 1931) & Palearctic & \\
\hline 70. & Microtydeus constans Thor, 1931 & Arctic & \\
\hline \multirow[b]{2}{*}{71.} & Sperchontidae & & \multirow{2}{*}{$\begin{array}{l}\text { Bjørnøya: Ellasjøen, Engelskelva, Hellevatnet, Mosevatnet, } \\
\text { Stevatnet }[91,99,111]\end{array}$} \\
\hline & Sperchon brevirostris (Koenike, 1895) & Holarctic & \\
\hline $\begin{array}{l}72 . \\
73 . \\
74 .\end{array}$ & $\begin{array}{c}\text { Trombidiidae } \\
\text { Podothrombium bicolor (Hermann, 1804) } \\
\text { P. curtipalpe (Thor, 1900)* } \\
\text { P. svalbardense Oudemans, } 1930^{*,+}\end{array}$ & $\begin{array}{l}\text { Palearctic } \\
\text { Holarctic } \\
\text { Arctic }\end{array}$ & $\begin{array}{l}\text { Spitsbergen: Hiorthhamn [91] } \\
\text { Spitsbergen: Hiorthhamn [91] } \\
\text { Spitsbergen: Hiorthhamn [91] }\end{array}$ \\
\hline 75. & $\begin{array}{c}\text { Syringophilidae } \\
\text { Chenophila nanseni Skoracki \& } \\
\text { Zawierucha } 2016^{*,+}\end{array}$ & Arctic & Spitsbergen: Nissenfjella [57] \\
\hline $\begin{array}{l}76 . \\
77 .\end{array}$ & $\begin{array}{c}\text { Stigmaeidae } \\
\text { Eustigmaeus oudemansi (Thor, 1930) } \\
\text { E. pulchellus (Thor, 1930) } \\
\text { *,t }\end{array}$ & $\begin{array}{l}\text { Arctic } \\
\text { Arctic }\end{array}$ & \multirow{4}{*}{$\begin{array}{l}\text { Spitsbergen: “Großer Trichter”, Tempelfjorden [103]; Svalbard } \\
\text { [106,112] } \\
\text { Bjørnøya [105]; Spitsbergen: Salmon Lake at Dirksbukta, south } \\
\text { side of Reinsdyrflya [7] }\end{array}$} \\
\hline \multirow[b]{2}{*}{78.} & Tetranychidae & & \\
\hline & Bryobia borealis Oudemans, 1930 * & Holarctic & \\
\hline 79. & B. praetiosa C.L. Koch 1836 & Cosmopolitan & \\
\hline \multirow[t]{3}{*}{80.} & $\begin{array}{c}\text { Pygmephoridae } \\
\text { Kerdabania arctica (Thor, 1934) }{ }^{4, *,+}\end{array}$ & Arctic & Spitsbergen: Templefjorden [103] \\
\hline & SARCOPTIFORMES & & \\
\hline & Endeostigmata & & \\
\hline
\end{tabular}


Table 1. Cont

\begin{tabular}{|c|c|c|c|}
\hline No. & Taxon & Distribution & Localities \\
\hline \multirow[b]{2}{*}{81.} & Nanorchestidae & & \\
\hline & Nanorchestes arboriger (Berlese, 1904) ${ }^{5}$ & Palearctic & $\begin{array}{c}\text { Spitsbergen: “Großer Trichter", Hiorthhamn, } \\
\text { Magdalenefjorden }[91,103]\end{array}$ \\
\hline & Alicorhagiidae & & \\
\hline 82. & Alicorhagia clavipilus (Thor, 1931)* & Arctic & Spitsbergen: “Großer Trichter”, Hiorthhamn $[103,113]$ \\
\hline 83. & A. plumipilis (Thor, 1931)* & Palearctic & Spitsbergen: “Großer Trichter” [103] \\
\hline \multirow{3}{*}{$\begin{array}{l}84 . \\
85 .\end{array}$} & \multirow{3}{*}{$\begin{array}{c}\text { Eriophyidae } \\
\text { Aceria saxifragae (Rostrup, 1900) } \\
\text { Cecidophyes siedleckii Kiedrowicz, Szydlo } \\
\text { \& Skoracka, 2016 }{ }^{*,+}\end{array}$} & & \\
\hline & & Holarctic & Spitsbergen: Hornsund [67] \\
\hline & & Arctic & Spitsbergen: Hornsund [67] \\
\hline \multicolumn{3}{|c|}{ Oribatida } & \\
\hline \multicolumn{3}{|c|}{ Brachychthoniidae } & \\
\hline 86. & Brachychthonius laetepictus Berlese, 1910 & Palaearctic & Spitsbergen: Magdalenefjorden, Pyramiden [20,103] \\
\hline 87. & Eobrachychthonius borealis Forsslund, 1942 & Holarctic & $\begin{array}{c}\text { Bjørnøya [83]; Spitsbergen: Barentsburg, Longyearbyen, } \\
\text { Recherchefjorden }[14,114]\end{array}$ \\
\hline 88. & E. latior (Berlese, 1910) & Holarctic & $\begin{array}{c}\text { Danskøya: Virgohamna [115]; Spitsbergen: Bockfjorden, } \\
\text { Fivelflyene, Flathuken, “Großer Trichter”, Helvetiadalen, } \\
\text { Hiorthhamn, Isdammen, Longyearbyen, Magdalenefjorden, } \\
\text { Reinsdyrflya, Sassendalen, Sven Olssonodden, Sørgattet } \\
{[91,103,115,116]}\end{array}$ \\
\hline 89. & E. oudemansi Hammen, 1952 & $\begin{array}{l}\text { Holarctic, Neotropical, Indomalayan, } \\
\text { Antarctic }\end{array}$ & $\begin{array}{c}\text { Edgeøya: Kapp Lee, Russebukta [8] } \\
\text { Spitsbergen: Longyearbyen, Pyramiden, Recherchefjorden } \\
{[20,114,116]}\end{array}$ \\
\hline 90. & Liochthonius alpestris (Forsslund, 1958) & Palearctic & Spitsbergen: Hornsund [88] \\
\hline 91. & L. brevis (Michael, 1888) & Holarctic & $\begin{array}{c}\text { Spitsbergen: Adventdalen, Barentsburg, Fivelflyene, Flathuken, } \\
\text { Hiorthhamn, Isdammen, Reinsdyrflya, Worsleyneset } \\
{[19,91,115,116]}\end{array}$ \\
\hline 92. & L. clavatus (Forsslund, 1942) & Palaearctic & Edgeøya: Blåbukta, Kapp Heuglin [8] \\
\hline & & & Bjørnøya [83]; Spitsbergen: Barentsburg, Hornsund, \\
\hline 93. & L. lapponicus (Trägårdh, 1910) & Holarctic & $\begin{array}{l}\text { Longyearbyen, Midtre Lovénbreen, Ny-Ålesund, } \\
\text { Recherchefjorden }[10,14,15,88,114,117,118]\end{array}$ \\
\hline 94. & L. muscorum Forsslund, 1964 & Palaearctic & Spitsbergen: Barentsburg, Hornsund $[19,88]$ \\
\hline 95. & L. neglectus Moritz, 1976 & Palearctic & $\begin{array}{l}\text { Spitsbergen: Kongsfjorden [119]; based on Hodkinson's } \\
\text { material stored in Liverpool [120] }\end{array}$ \\
\hline
\end{tabular}


Table 1. Cont

\begin{tabular}{|c|c|c|c|}
\hline No. & Taxon & Distribution & Localities \\
\hline 96. & \multirow{4}{*}{$\begin{array}{l}\text { L. strenzkei Forsslund, } 1963 \\
\text { L. tuxeni (Forsslund, 1957) } \\
\text { Neoliochthonius piluliferus (Forsslund, } \\
\text { 1942) }\end{array}$} & Holarctic & $\begin{array}{c}\text { Bjørnøya [83]; Hopen: Koefoedodden, Hopen radio [86]; Spitsbergen: } \\
\text { Barentsburg, "Großer Trichter", Hiorthhamn, Longyearbyen, } \\
\text { Magdalenefjorden, Petuniabukta, Pyramiden, Recherchefjorden, } \\
\text { Vestpynten }[14,19,20,22,91,103,105,114]\end{array}$ \\
\hline 97. & & Holarctic & $\begin{array}{c}\text { Edgeøya: Blåbukta, Kapp Heuglin, Kapp Lee, Negerdalen, } \\
\text { Russebukta [8] }\end{array}$ \\
\hline 98. & & Palaearctic & Spitsbergen: Midtre Lovénbreen [10] \\
\hline 99. & & Holarctic & Edgeøya: Kapp Heuglin [8] \\
\hline 100. & $\begin{array}{l}\text { Eniochthoniidae } \\
\text { Eniochthonius minutissimus (Berlese, 1903) }\end{array}$ & Cosmopolitan & Spitsbergen: Petuniabukta [22] \\
\hline 101. & $\begin{array}{c}\text { Phthiracaridae } \\
\text { Atropacarus striculus (C.L. Koch, 1835) }\end{array}$ & $\begin{array}{l}\text { Holarctic, Oriental, Neotropical, } \\
\text { Australian }\end{array}$ & $\begin{array}{l}\text { Spitsbergen: Barentsburg, Grønfjordenbreen, Isfjorden, } \\
\text { Longyearbyen, Midtre Lovénbreen }[10,13,14,16]\end{array}$ \\
\hline 102. & Camisia anomia Colloff, 1993 & Arctic & $\begin{array}{c}\text { Bjørnøya [83]; Spitsbergen: Endalen, Kolhaugen, Kongsfjorden } \\
\text { [121-129] }\end{array}$ \\
\hline 103. & C. biverrucata (C.L. Koch, 1839) & Holarctic & $\begin{array}{l}\text { Edgeøya: Blåbukta, Kapp Heuglin [8]; Spitsbergen: Longyearbyen } \\
\text { [103] }\end{array}$ \\
\hline 104. & C. borealis (Thorell, 1871)* & Holarctic & $\begin{array}{l}\text { Danskøya: Virgohamna [115]; Spitsbergen: Barentsburg, Hornsund, } \\
\text { Isfjorden, Longyearbyen, Recherchefjorden }[14,16,102,114,115,130]\end{array}$ \\
\hline 105. & C. dictyna Colloff, 1993 & Holarctic & $\begin{array}{l}\text { Svalbard [71]; Spitsbergen: Midtre Lovénbreen [10] } \\
\text { Bjørnøya [83]; Edgeøya: Blåbukta, Kapp Heuglin, Negerdalen, }\end{array}$ \\
\hline 106. & C. foveolata Hammer, 1955 & Holarctic & $\begin{array}{l}\text { Russebukta [8]; Hopen: Hopen radio, Koefoedodden [86]; } \\
\text { Spitsbergen: Aldegondabreen, Grønfjordenbreen, Hornsund, } \\
\text { Kiærstranda, Knudsenheia, Midtre Lovénbreen [10,88,109,131] }\end{array}$ \\
\hline 107. & C. horrida (Hermann, 1804) & $\begin{array}{l}\text { Holarctic, Oriental, Ethiopian, } \\
\text { Neotropical }\end{array}$ & $\begin{array}{c}\text { Bjørnøya [91]; Edgeøya: Diskobukta, Kapp Lee, Russebukta [8]; } \\
\text { Spitsbergen: Barentsburg, “Großer Trichter”, Hiorthhamn, } \\
\text { Hiorthfjellet, Longyearbyen, Magdalenefjorden, Kongsfjorden, } \\
\text { Reinsdyrflya, Recherchefjorden, Vestpynten, Petuniabukta, } \\
\text { Pyramiden }[20,22,91,103,105,114,115,132]\end{array}$ \\
\hline 108. & C. invenusta (Michael, 1888) & Palaearctic & Svalbard [71] \\
\hline 109. & C. lapponica (Trägårdh, 1910) & Holarctic & Spitsbergen: Barentsburg, Hornsund, Longyearbyen $[14,16,88]$ \\
\hline 110. & C. spinifer (C.L. Koch, 1836) & Holarctic, Oriental, Neotropical & Spitsbergen: Tempelfjorden [103] \\
\hline 111. & Capillonothrus capillatus (Berlese, 1914) & Holarctic & Spitsbergen: Arctowskifjellet [115] \\
\hline 112. & Platynothrus peltifer (C.L. Koch, 1839) & Holarctic, Oriental, Australian & Spitsbergen: Recherchefjorden, Sassendalen $[114,115]$ \\
\hline 113. & P. punctatus (L. Koch, 1879) & Palaearctic & $\begin{array}{l}\text { Spitsbergen: Barentsburg, Grønfjordenbreen, Isfjorden, } \\
\text { Longyearbyen, Petuniabukta, Vestpynten }[13,14,16,22]\end{array}$ \\
\hline
\end{tabular}


Table 1. Cont

\begin{tabular}{|c|c|c|c|}
\hline No. & Taxon & Distribution & Localities \\
\hline \multicolumn{4}{|c|}{ Hermanniidae } \\
\hline 114. & Hermannia reticulata Thorell, 1871 * & Holarctic & $\begin{array}{l}\text { Bjørnøya: Fugleodden, Tunheim [83,91,105,133]; Edgeøya: } \\
\text { Blåbukta, Russebukta [134]; Prins Karls Forland: Silene Hill } \\
\text { [99,101]; Spitsbergen: Barentsburg, Bellsund, Endalen, “Großer } \\
\text { Trichter”, Kongsfjorden, Longyearbyen, Magdalenefjorden, }\end{array}$ \\
\hline & & (20) & $\begin{array}{l}\text { Midtre Lovénbreen, Ny-Ålesund, Observasjonsholmen, } \\
\text { Recherchefjorden, Petuniabukta, Tempelfjorden, Vestpynten } \\
{[10,12,15,22,91,102,103,114,121-123,125,129,132-134]}\end{array}$ \\
\hline 115. & H. scabra (L. Koch, 1879) & Holarctic & $\begin{array}{c}\text { Bjørnøya [83,105]; Edgeøya: Kapp Lee [135]; Prins Karls } \\
\text { Forland: Fuglehuken fyr [135]; Spitsbergen: Kapp Laila, } \\
\text { Petuniabukta [135] }\end{array}$ \\
\hline 116. & $\begin{array}{c}\text { Malaconothridae } \\
\text { Malaconothrus monodactylus (Michael, } \\
\text { 1888) }\end{array}$ & Palaearctic & $\begin{array}{c}\text { Spitsbergen: Barentsburg, Grønfjordenbreen, Isfjorden, } \\
\text { Longyearbyen }[13,14,16]\end{array}$ \\
\hline 117. & $\begin{array}{c}\text { Nanhermanniidae } \\
\text { Nanhermannia sellnicki Forsslund, } 1958\end{array}$ & Palearctic & Spitsbergen: Barentsburg, Longyearbyen [14] \\
\hline \multicolumn{4}{|c|}{ Nothridae } \\
\hline 118. & Nothrus palustris C.L. Koch, 1839 & Holarctic & $\begin{array}{c}\text { Spitsbergen: Barentsburg, Grønfjordenbreen, Isfjorden, } \\
\text { Longyearbyen }[13,16]\end{array}$ \\
\hline \multicolumn{3}{|c|}{ Damaeidae } & Spitsbergen: Barentsburg, Longyearbyen [16] \\
\hline 120. & $\begin{array}{l}\text { Kunstidamaeus arcticus Miko \& Monson, } \\
\qquad 2013^{*,+}\end{array}$ & Svalbard & Bjørnøya [83]; Spitsbergen [72] \\
\hline 121. & Subbelba montana (Kulczynski, 1902) & Palearctic & Bjørnøya: Austervåg [91] \\
\hline \multicolumn{4}{|c|}{ Peloppiidae } \\
\hline 122. & Ceratoppia bipilis (Hermann, 1804) & Holarctic, Oriental, Neotropical & $\begin{array}{c}\text { Prins Karls Forland: Silene Hill [99,101]; Spitsbergen: } \\
\text { Kongsfjorden, Storholmen [7,12] }\end{array}$ \\
\hline 123. & C. hoeli Thor, 1930 * & Arctic & $\begin{array}{l}\text { Bjørnøya: Tunheim [91]; Spitsbergen: Barentsburg, Bockfjorden, } \\
\text { "Großer Trichter", Grønfjordenen, Hiorthfjellet, Hiorthhamn, } \\
\text { Isdammen, Kolhaugen, Kongsfjorden, Longyearbyen, } \\
\text { Magdalenefjorden, Recherchefjorden, Worsleyneset } \\
\text { [91,103,114,115,121-125,127] }\end{array}$ \\
\hline 124. & C. sphaerica (L. Koch, 1879) & Palaearctic & $\begin{array}{c}\text { Bjørnøya [83]; Edgeøya: Kapp Lee, Russebukta [8]; Hopen: } \\
\text { Hopen radio, Koefoedodden [86]; Spitsbergen: Barentsburg, } \\
\text { Hornsund, Longyearbyen, Petuniabukta, Pyramiden, } \\
\text { Vestpynten [14-16,20,22,130] }\end{array}$ \\
\hline
\end{tabular}


Table 1. Cont.

\begin{tabular}{|c|c|c|c|}
\hline No. & Taxon & Distribution & Localities \\
\hline $\begin{array}{l}125 . \\
126 .\end{array}$ & $\begin{array}{c}\text { Carabodidae } \\
\text { Carabodes labyrinthicus (Michael, 1879) } \\
\text { C. marginatus (Michael, 1884) }\end{array}$ & $\begin{array}{l}\text { Holarctic } \\
\text { Palearctic }\end{array}$ & $\begin{array}{l}\text { Spitsbergen: Barentsburg, Longyearbyen [14] } \\
\text { Spitsbergen: Barentsburg, Longyearbyen [16] }\end{array}$ \\
\hline $\begin{array}{l}127 . \\
128\end{array}$ & $\begin{array}{c}\text { Autognetidae } \\
\text { Autogneta kaisilai Karppinen, 1967* } \\
\text { Conchogneta dalecarlica (Forsslund, 1947) }\end{array}$ & $\begin{array}{c}\text { Arctic } \\
\text { Palearctic }\end{array}$ & $\begin{array}{l}\text { Spitsbergen: Biskayerhuken [115] } \\
\text { Spitsbergen: Midtre Lovénbreen [10] }\end{array}$ \\
\hline 129. & Dissorhina ornata (Oudemans, 1900) & Holarctic & $\begin{array}{l}\text { Bjørnøya: Fugleodden, Mosevatnet [91]; Spitsbergen: } \\
\text { Adventdalen, Barentsburg, Biskayerhuken, Fivelflyene, } \\
\text { Hiorthhamn, Isdammen, Longyearbyen, Magdalenefjorden, } \\
\text { Petuniabukta, Pyramiden }[14,20,22,91,103,115]\end{array}$ \\
\hline 130. & Lauroppia fallax (Paoli, 1908) & $\begin{array}{l}\text { Holarctic, IndoMalayan, Australian, } \\
\text { Neotropical }\end{array}$ & Spitsbergen: Magdalenefjorden [103] \\
\hline 131. & Microppia minus (Paoli, 1908) & Cosmopolitan & Spitsbergen: Hornsund [136] \\
\hline 132. & $\begin{array}{l}\text { Moritziella microdentata Gordeeva \& } \\
\text { Grishina, } 1991\end{array}$ & Palearctic & Spitsbergen: Hornsund [137] \\
\hline 133. & Moritzoppia splendens (C.L. Koch, 1841) & Holarctic & $\begin{array}{c}\text { Bjørnøya [83]; Spitsbergen: Barentsburg, Grønfjordenbreen, } \\
\text { Isfjorden, Longyearbyen }[13,14,16]\end{array}$ \\
\hline 134. & M. unicarinata (Paoli, 1908) & Holarctic & $\begin{array}{c}\text { Edgeøya: Negerdalen [8]; Spitsbergen: Barentsburg, } \\
\text { Longyearbyen [14,16] }\end{array}$ \\
\hline 135. & Oppiella neerlandica (Oudemans, 1900) & Holarctic & $\begin{array}{c}\text { Edgeøya: Kapp Heuglin [8]; Spitsbergen: Barentsburg, “Großer } \\
\text { Trichter", Hanaskogdalen, Hiorthhamn, Kongsfjord, } \\
\text { Longyearbyen, Magdalenefjorden, Midtre Lovénbreen, } \\
\text { Vestpynten }[10,14,19,22,91,103,125]\end{array}$ \\
\hline 136. & O. nova (Oudemans, 1902) & Cosmopolitan & $\begin{array}{c}\text { Spitsbergen: Adventdalen, Barentsburg, Grønfjordenbreen, } \\
\text { Hornsund, Isdammen, Isfjorden, Kongsfjorden, Longyearbyen, } \\
\text { Petuniabukta, Reinsdyrflya, Worsleyneset } \\
{[13,14,16,19,22,115,121,123,136]}\end{array}$ \\
\hline 137. & O. translamellata (Willmann, 1923) & Holarctic & $\begin{array}{l}\text { Edgeøya: Diskobukta, Kapp Lee, Russebukta [8]; Hopen: } \\
\text { Hopen radio, Koefoedodden [84]; Spitsbergen: Adventdalen, } \\
\text { Arctowskifjellet, Barentsburg, Bockfjorden, Fivelflyene, } \\
\text { Grønfjordenbreen, Hornsund, Isfjorden, Kolhaugen, } \\
\text { Longyearbyen, Magdalenefjorden, Petuniabukta, } \\
\text { Recherchefjorden, Reinsdyrflya, Stuphallet, Vestpynten, } \\
\text { Worsleyneset }[13,14,16,22,103,114,115,124,136,138]\end{array}$ \\
\hline
\end{tabular}


Table 1. Cont

\begin{tabular}{|c|c|c|c|}
\hline No. & Taxon & Distribution & Localities \\
\hline \multicolumn{4}{|c|}{ Suctobelbidae } \\
\hline 138. & Suctobelba hammerae Krivolutsky, 1965 & Holarctic & $\begin{array}{c}\text { Spitsbergen: Barentsburg, Grønfjordenbreen, Isfjorden, } \\
\text { Longyearbyen }[13,14,16]\end{array}$ \\
\hline 139. & Suctobelbella sarekensis (Forsslund, 1941) & Holarctic & Spitsbergen: Adventdalen, Fivelflyene [115] \\
\hline 140. & S. subcornigera (Forsslund, 1941) & Holarctic, Oriental, Australian & $\begin{array}{c}\text { Spitsbergen: Barentsburg, Biskayerhuken, Grønfjordenbreen, } \\
\text { Isfjorden, Longyearbyen, Reinsdyrflya, Worsleyneset } \\
{[13,14,16,22,115]}\end{array}$ \\
\hline & Tectocepheidae & & \multirow{5}{*}{$\begin{array}{c}\text { Spitsbergen: Recherchefjorden [114] } \\
\text { Spitsbergen: Barentsburg, Longyearbyen [14,16] } \\
\text { Spitsbergen: Adventdalen, Blomstrandhalvøya, Bockfjorden, } \\
\text { Isdammen, Kongsfjorden, Reinsdyrflya, Worsleyneset [115] } \\
\text { Bjørnøya [83]; Edgeøya: Diskobukta, Kapp Lee, Russebukta [8]; } \\
\text { Spitsbergen: Barentsburg, Hiorthhamn, Kolhaugen, } \\
\text { Kongsfjorden, Longyearbyen, Midtre Lovénbreen, } \\
\text { Petuniabukta, Pyramiden, Vestpynten } \\
\text { [10,14,16,19,20,22,91,103,105,124,125] }\end{array}$} \\
\hline 141. & Tectocepheus alatus Berlese, 1913 & Palearctic & \\
\hline 142. & T. knuellei Vanek, 1960 & Palearctic & \\
\hline 143. & T. sarekensis Trägårdh, 1910 & Palearctic & \\
\hline 144. & T. velatus (Michael, 1880) & Cosmopolitan & \\
\hline \multicolumn{4}{|c|}{ Ameronothridae } \\
\hline 145. & Ameronothrus lineatus (Thorell, 1871)* & Palaearctic & $\begin{array}{c}\text { Bjørnøya: Engelskelva, Fugleodden, Tunheim } \\
\text { [83,88,99,101,103,105,132,133,139]; Lågøya: [7]; Prins Karls } \\
\text { Forland: MacKenziedale, Richardlaguna almost to } \\
\text { Carmichaelpynten [99,101]; Spitsbergen: Adventdalen, } \\
\text { Barentsburg, Bockfjorden, Colesbukta, Grønfjordenbreen, }\end{array}$ \\
\hline & & & $\begin{array}{l}\text { Kolhaugen, Liefdefjorden, Nordenskiöld Land, Ny-Ålesund, } \\
\text { Observasjonsholmen, Reinsdyrflya, Smeerenburg, } \\
\text { Worsleyneset [7,12,91,102,109,115,124,132,133,140-145] }\end{array}$ \\
\hline 146. & A. nidicola Sitnikova, 1975 & Palaearctic & Spitsbergen: Hornsund [88] \\
\hline 147. & A. nigrofemoratus (L. Koch, 1879) & Palearctic & Bjørnøya [83] \\
\hline 148. & $\begin{array}{c}\text { Micreremidae } \\
\text { Micreremus brevipes (Michael, 1888) }\end{array}$ & Palearctic & Spitsbergen: Recherchefjorden [114] \\
\hline 149. & $\begin{array}{c}\text { Scutoverticidae } \\
\text { Scutovertex minutus (C.L. Koch, 1835) }\end{array}$ & Palearctic & Spitsbergen: Bockfjorden, Recherchefjorden $[114,115]$ \\
\hline \multicolumn{4}{|c|}{ Achipteriidae } \\
\hline 150. & Achipteria punctata (Nicolet, 1855) & Holarctic & $\begin{array}{l}\text { Spitsbergen: Barentsburg, Grønfjordenbreen, Isfjorden, } \\
\text { Longyearbyen, Vestpynten }[13,14,16,22]\end{array}$ \\
\hline
\end{tabular}


Table 1. Cont

\begin{tabular}{|c|c|c|c|}
\hline No. & Taxon & Distribution & Localities \\
\hline 151. & $\begin{array}{c}\text { Tegoribatidae } \\
\text { Scutozetes clavatosensillus Ermilov, } \\
\text { Martens \& Tolstikov, } 2013\end{array}$ & Palearctic & Spitsbergen: Mosselbukta [146] \\
\hline \multirow[t]{2}{*}{152.} & $\begin{array}{c}\text { Oribatellidae } \\
\text { Oribatella arctica Thor, } 1930 \text { * }\end{array}$ & Holarctic & $\begin{array}{l}\text { Edgeøya: Diskobukta, Kapp Lee [8,147]; Spitsbergen: } \\
\text { Barentsburg, Hiorthhamn, Longyearbyen, Mälardalen, } \\
\text { Petuniabukta, Recherchefjorden }[15,22,91,103,114]\end{array}$ \\
\hline & Oribatulidae & & \\
\hline 153. & Oribatula exilis (Nicolet, 1855) & Holarctic & $\begin{array}{c}\text { Bjørnøya: Fugleodden, Mosevatnet, Tunheim [91]; Prins Karls } \\
\text { Forland: Silene Hill [99,101]; Spitsbergen: Adventdalen, } \\
\text { Barentsburg, Engelskhytta, Hanaskogdalen, Hiorthhamn, } \\
\text { Isfjord radio, Longyearbyen }[14,91]\end{array}$ \\
\hline 154. & O. tibialis (Nicolet, 1855) & Holarctic & $\begin{array}{l}\text { Bjørnøya [83]; Edgeøya: Diskobukta, Kapp Lee, Russebukta [8]; } \\
\text { Spitsbergen: Barentsburg, Longyearbyen, Observasjonsholmen, } \\
\text { Petuniabukta, Storholmen, Vestpynten [12,14,15,19,22] } \\
\text { Bjørnøya [83]; Spitsbergen: Adventdalen, Fivelflyene, “Großer }\end{array}$ \\
\hline 155. & O. venusta Berlese, 1908 & Holarctic & $\begin{array}{c}\text { Trichter", Longyearbyen, Magdalenefjorden, Recherchefjorden, } \\
\text { Reinsdyrflya, Worsleyneset }[103,114,115]\end{array}$ \\
\hline \multirow[t]{2}{*}{156.} & Phauloppia lucorum (C.L. Koch, 1841) & Palearctic & Spitsbergen: Bellsund, Isfjorden $[81,133]$ \\
\hline & Scheloribatidae & & \\
\hline \multirow[t]{2}{*}{157.} & Liebstadia similis (Michael, 1888) & Holarctic, Indomalayan, Australian & $\begin{array}{c}\text { Spitsbergen: Barentsburg, Grønfjordenbreen, Isfjorden, } \\
\text { Longyearbyen }[13,14,16]\end{array}$ \\
\hline & Ceratozetidae & & \\
\hline 158. & Ceratozetes spitsbergensis Thor, 1934 * & Palearctic & $\begin{array}{l}\text { Edgeøya: Blåbukta, Kapp Heuglin, Negerdalen [8]; Kong Karls } \\
\text { Land [148]; Spitsbergen: Arctowskifjellet, Barentsburg, “Großer } \\
\text { Trichter”, Kolhaugen, Longyearbyen, Magdalenefjorden, } \\
\text { Pyramiden, Reinsdyrflya, Worsleyneset [14,20,103,115,124] }\end{array}$ \\
\hline
\end{tabular}


Table 1. Cont.

\begin{tabular}{|c|c|c|c|}
\hline No. & Taxon & Distribution & Localities \\
\hline 159. & Diapterobates notatus (Thorell, 1871) * & Holarctic & $\begin{array}{c}\text { Bjørnøya: Fugleodden, Tunheim [14-16,83,91,99,101,105,133]; } \\
\text { Barentsøya [130]; Edgeøya: Blåbukta, Diskobukta, Kapp } \\
\text { Heuglin, Kapp Lee, Negerdalen, Russebukta, Tjuvfjorden } \\
\text { [8,146]; Hopen: Hopen radio, Koefoedodden [86]; Prins Karls } \\
\text { Forland: Fuglehuken, MacKenziedale, Silene Hill [99,101,146]; } \\
\text { Sofiaøya [146]; Spitsbergen: Adventfjorden, Barentsburg, } \\
\text { Bellsund, Billefjorden, Blomstrandhalvøya, Bohemanneset, } \\
\text { Dirksbukta, Endalen, Engelskhytta, “Großer Trichter", } \\
\text { Grønfjordenen, Hiorthfjellet, Hiorthhamn, Hornsund, Isfjorden, } \\
\text { Kolhaugen, Krykkjefjellet, Liefdefjorden, Longyearbyen, } \\
\text { Magdalenefjorden, Midtre Lovénbreen, Ossian Sarsfjellet, } \\
\text { Petuniabukta, Recherchefjorden, Reinsdyrflya, Sven } \\
\text { Olssonodden, Storholmen, Vestpynten, Worsleyneset } \\
\text { [7,10,12,14-17,19,22,91,99,101-103,114,115,121-125,129,130,132, } \\
133,146,148]\end{array}$ \\
\hline 160. & Edwardzetes edwardsi (Nicolet, 1855) & Holarctic & $\begin{array}{l}\text { Spitsbergen: Tempelfjorden; possibly from the North Cape } \\
\text { [103] }\end{array}$ \\
\hline 161. & 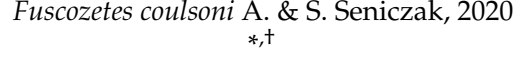 & Arctic & Edgeøya: Negerdalen [9] \\
\hline 162. & Iugoribates gracilis Sellnick, 1944 & Holarctic & $\begin{array}{l}\text { Spitsbergen: Kongsfjorden [119]; based on Hodkinson's } \\
\text { material stored in Liverpool [120] }\end{array}$ \\
\hline 163. & Oromurcia lucens (L. Koch, 1879) & Holarctic & Barentsøya [130]; Spitsbergen: Vestpynten [22] \\
\hline 164. & Svalbardia paludicola Thor, 1930 * & Holarctic & $\begin{array}{l}\text { Spitsbergen: Hanaskogdalen, Hiorthhamn, Hornsund, } \\
\text { Reinsdyrflya, Ringhorndalen, Sørkapp }[91,139,146]\end{array}$ \\
\hline 165. & Trichoribates berlesei Jacot, 1929 & Holarctic & Spitsbergen: Hornsund, Storholmen $[12,88]$ \\
\hline 166. & T. novus (Sellnick, 1928) & Holarctic & Spitsbergen: Hornsund [88] \\
\hline 167. & T. setiger (Trägårdh, 1910) & Palaearctic & Bjørnøya [83]; Spitsbergen: Recherchefjorden [114] \\
\hline $\begin{array}{l}168 . \\
169 .\end{array}$ & $\begin{array}{c}\text { Chamobatidae } \\
\text { Chamobates birulai (Kulczynski, 1902)* } \\
\text { C. borealis (Trägårdh, 1902) }\end{array}$ & $\begin{array}{l}\text { Palaearctic } \\
\text { Palaearctic }\end{array}$ & $\begin{array}{l}\text { Spitsbergen [133] } \\
\text { Spitsbergen: Hornsund [88] }\end{array}$ \\
\hline $\begin{array}{l}170 . \\
171 .\end{array}$ & $\begin{array}{c}\text { Punctoribatidae } \\
\text { Mycobates bicornis (Strenzke, 1954) } \\
\text { M. parmeliae (Michael, 1884) }\end{array}$ & $\begin{array}{l}\text { Palaearctic } \\
\text { Holarctic }\end{array}$ & $\begin{array}{c}\text { Spitsbergen: Midtre Lovénbreen [10] } \\
\text { Spitsbergen: Barentsburg, Longyearbyen, Midtre Lovénbreen } \\
\qquad[10,14,16]\end{array}$ \\
\hline
\end{tabular}


Table 1. Cont

\begin{tabular}{|c|c|c|c|}
\hline No. & Taxon & Distribution & Localities \\
\hline 172. & M. sarekensis (Trägårdh, 1910) & Holarctic & $\begin{array}{l}\text { Bjørnøya [91]; Edgeøya: Kapp Lee, Russebukta [8,149]; } \\
\text { Spitsbergen: Adventdalen, Arctowskifjellet, Bockfjorden, } \\
\text { Engelskhytta, Fjortende Julibukta, “Großer Trichter”, } \\
\text { Hiorthfjellet, Hiorthhamn, Hornsund, Isdammen, } \\
\text { Kongsfjorden, Longyearbyen, Magdalenefjorden, Mosselbukta, } \\
\text { Recherchefjorden, Sassendalen, Signehamna, Storholmen, } \\
\text { Sørkapp, Vestpynten [22,91,103,114,115,121,123,125,149] }\end{array}$ \\
\hline 173. & M. tridactylus Willmann, 1929 & Holarctic & Spitsbergen: Barentsburg, Longyearbyen [14] \\
\hline 174. & Punctoribates punctum (C.L. Koch, 1839) & Holarctic, Oriental, Australian & $\begin{array}{l}\text { Spitsbergen: Barentsburg, Longyearbyen, Petuniabukta } \\
{[114,16,22]}\end{array}$ \\
\hline 175. & $\begin{array}{c}\text { Galumnidae } \\
\text { Pergalumna nervosa (Berlese, 1914) }\end{array}$ & Holarctic & Spitsbergen: Hornsund [136] \\
\hline 176. & $\begin{array}{c}\text { Acaridae } \\
\text { Sancassania mycophagus (Mégnin, 1874) }\end{array}$ & Cosmopolitan & Spitsbergen: Hornsund [91] \\
\hline 177. & $\begin{array}{c}\text { Alloptidae } \\
\text { Alloptes (Sternalloptes) stercorarii Dubinin, } \\
1952\end{array}$ & Arctic & Spitsbergen: Ny-Å̀lesund [74] \\
\hline 178. & $\begin{array}{c}\text { Avenzoariidae } \\
\text { Zachvatkinia isolata Mironov, } 1989\end{array}$ & $\begin{array}{l}\text { Arctic, Neotropical, Australian, } \\
\text { Afrotropical }\end{array}$ & Spitsbergen: Ny-Å̀lesund [74] \\
\hline
\end{tabular}


$\mathbf{A}$

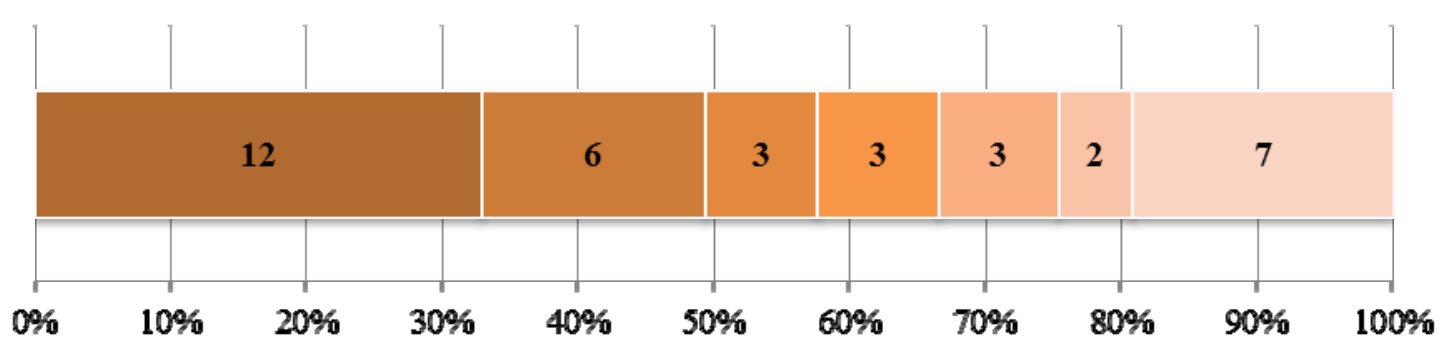

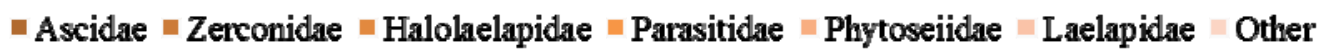

B

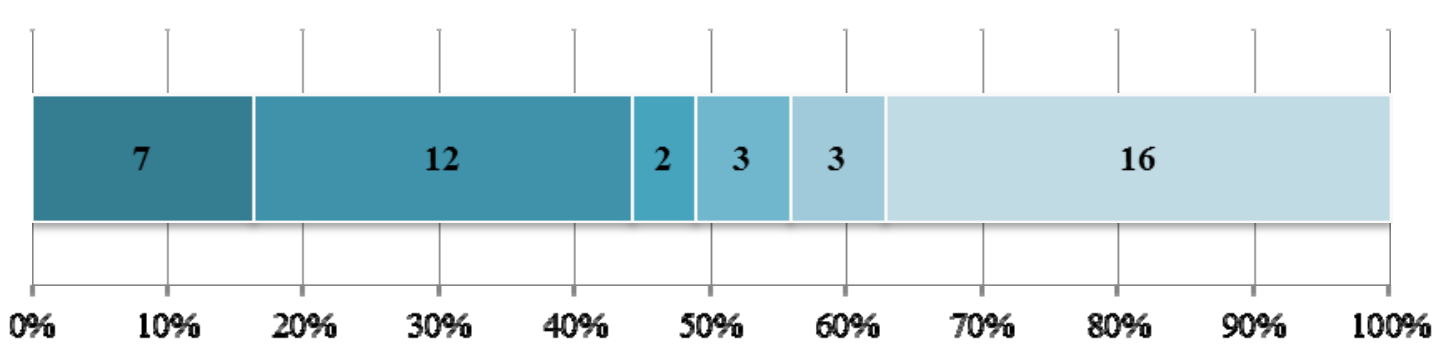

- Bdellidae $=$ Halacaridae $=$ Tydeidae $\|$ Iolinidae $\|$ Trombididae $\approx$ Other

C

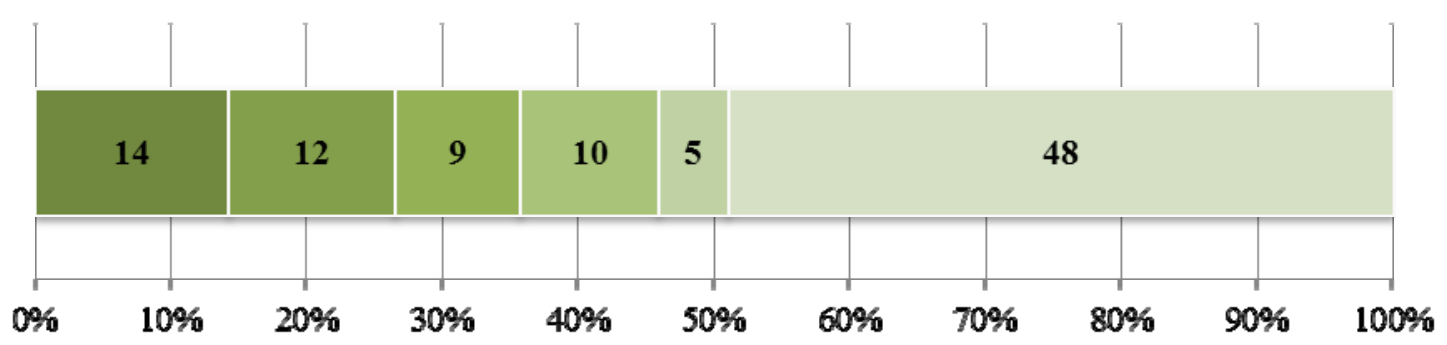

- Brachychthoniidae $=$ Crotoniidae $=$ Oppiidae $=$ Ceratozetidae $\backsim$ Punctoribatidae $\approx$ Other

Figure 2. Diversity of the mite families in Svalbard: (A) Mesostigmata; (B) Trombidiformes; (C) Sarcoptiformes. The number of species occurring in Svalbard is presented on bars. No figure was made for the Ixodida, which is represented in Svalbard by one family only.

More than a half of the Mesostigmata species known from Svalbard have been recorded there only once (Table 2). The majority of these are recent findings, but four were reported only in the first half of the last century, including Arctoseius laterincisus, which has an Arctic distribution. Four other records come from the second half of the last century, including another species with an Arctic distribution-Halolaelaps gerlachi. 
Table 2. Mite species found on Svalbard only once (or by the same author at a similar time).

\begin{tabular}{|c|c|c|c|}
\hline Until 1950 & 1951-2000 & After 2001 & Reference \\
\hline \multicolumn{4}{|l|}{ Mesostigmata } \\
\hline \multirow[t]{2}{*}{ U. acuminatus } & & & [81] \\
\hline & & Z. curiosus & [85] \\
\hline \multirow[t]{8}{*}{ Z. triangularis } & & & [91] \\
\hline & & P. (A.) insertus & [18] \\
\hline & & V. immanis & [92] \\
\hline & & V. remberti & [18] \\
\hline & & D. foveolatus & [20] \\
\hline & & H. coulsoni * & [93] \\
\hline & H. gerlachi* & & [94] \\
\hline & & S. baloghi & [8] \\
\hline \multirow[t]{3}{*}{ G. borealis } & & & [7] \\
\hline & & M. muscaedomesticae & [84] \\
\hline & A. cetratus & & [96] \\
\hline \multirow[t]{4}{*}{ A. laterincisus * } & & & [91] \\
\hline & A. ornatus & & [96] \\
\hline & & N. grumantensis * & [28] \\
\hline & L. hilaris & & [100] \\
\hline \multicolumn{4}{|l|}{ Trombidiformes } \\
\hline B. semiscutata* & & & {$[91,103]$} \\
\hline N. capillatus & & & [91] \\
\hline \multirow[t]{2}{*}{ C. croceus } & & & [103] \\
\hline & & A. saxifragae & [67] \\
\hline P. borneri * & & & [103] \\
\hline P. maior & & & [102] \\
\hline C. clavifrons & & & {$[91,103]$} \\
\hline B. alberti* & & & [107] \\
\hline C. pouchet ${ }^{*}$ & & & [81] \\
\hline C. reticulatus ${ }^{*}$ & & & [107] \\
\hline \multirow[t]{3}{*}{ C. richardi ${ }^{*}$} & & & [107] \\
\hline & & H. subterraneus & [45] \\
\hline & & H. subcrispus & [45] \\
\hline \multirow[t]{4}{*}{ H. borealis * } & & & [81] \\
\hline & & I. levis & [109] \\
\hline & & R. spinipes & [109] \\
\hline & & R. subtilis & [45] \\
\hline T. coeca* & & & [107] \\
\hline T. princeps * & & & [107] \\
\hline T. globifer * & & & [103] \\
\hline T. tenuiclaviger * & & & [103] \\
\hline M. constans * & & & [103] \\
\hline T. langei * & & & [103] \\
\hline P. bicolor & & & [91] \\
\hline P. curtipalpe* & & & [91] \\
\hline P. sualbardense * & & & [91] \\
\hline E. oudemansi * & & & [91] \\
\hline E. pulchellus * & & & [91] \\
\hline & & C. nanseni* ${ }^{*}$ & [57] \\
\hline K. arctica* & & & [103] \\
\hline
\end{tabular}


Table 2. Cont.

\begin{tabular}{|c|c|c|c|}
\hline Until 1950 & 1951-2000 & After 2001 & Reference \\
\hline \multicolumn{4}{|l|}{ Sarcoptiformes } \\
\hline A. clavipilus* & & & {$[103,113]$} \\
\hline \multirow[t]{6}{*}{ A. plumipilis * } & & & [103] \\
\hline & & C. siedleckii * & [67] \\
\hline & L. alpestris & & [88] \\
\hline & & L. clavatus & [8] \\
\hline & & L. neglectus & [119] \\
\hline & & L.tuxeni & [10] \\
\hline \multirow[t]{4}{*}{ C. spinifer } & & & [103] \\
\hline & C. capillatus & & [115] \\
\hline & & N. sellnicki & [14] \\
\hline & & D. onustus & [16] \\
\hline \multirow[t]{5}{*}{ S. montana } & & & [91] \\
\hline & & C. labyrinthicus & [14] \\
\hline & & C. marginatus & [16] \\
\hline & A. kaisilai * & & [115] \\
\hline & & C. dalecarlica & [10] \\
\hline \multirow[t]{8}{*}{ L. fallax } & & & [103] \\
\hline & M. minus & & [136] \\
\hline & S. sarekensis & & [115] \\
\hline & T. alatus & & [114] \\
\hline & T. sarekensis & & [115] \\
\hline & A. nidicola & & [88] \\
\hline & & A. nigrofemoratus & [83] \\
\hline & & S. clavatosensillus & [146] \\
\hline \multirow[t]{8}{*}{ E. edwardsi } & & & [103] \\
\hline & & F. coulsoni * & [9] \\
\hline & & I. gracilis & [119] \\
\hline & T. novus & & [88] \\
\hline & C. birulai ${ }^{*}$ & & [133] \\
\hline & C. borealis & & [88] \\
\hline & & M. bicornis & [10] \\
\hline & P. nervosa & & [136] \\
\hline \multirow[t]{3}{*}{ S. mycophagus } & & & [91] \\
\hline & & A. stercorarii & [74] \\
\hline & & Z. isolata & [74] \\
\hline
\end{tabular}

Note: *-new to science.

Most of the Mesostigmata species known from Svalbard have a Palaearctic distribution (Figure 3). Arctic species are also abundant and comprise nearly $40 \%$ of Svalbards total mesostigmatic diversity, while Holarctic species are less numerous. Five species have so far been found only in Svalbard (Table 1), including recent records (Halolaelaps coulsoni and Neoseiulus grumantensis) and historic records (Halolaelaps gerlachi, Arctoseius laterincisus and Neoseiulus magnanalis). Mesostigmata have been recorded from five islands in the Svalbard archipelago (Figure 1). Although most of the species are known from Spitsbergen, Saprosecans baloghi Karg, 1964, is known only from Edgeøya and Zerconopsis muestairi (Schweizer, 1949) has been found exclusively on Bjørnøya (Table 1). 

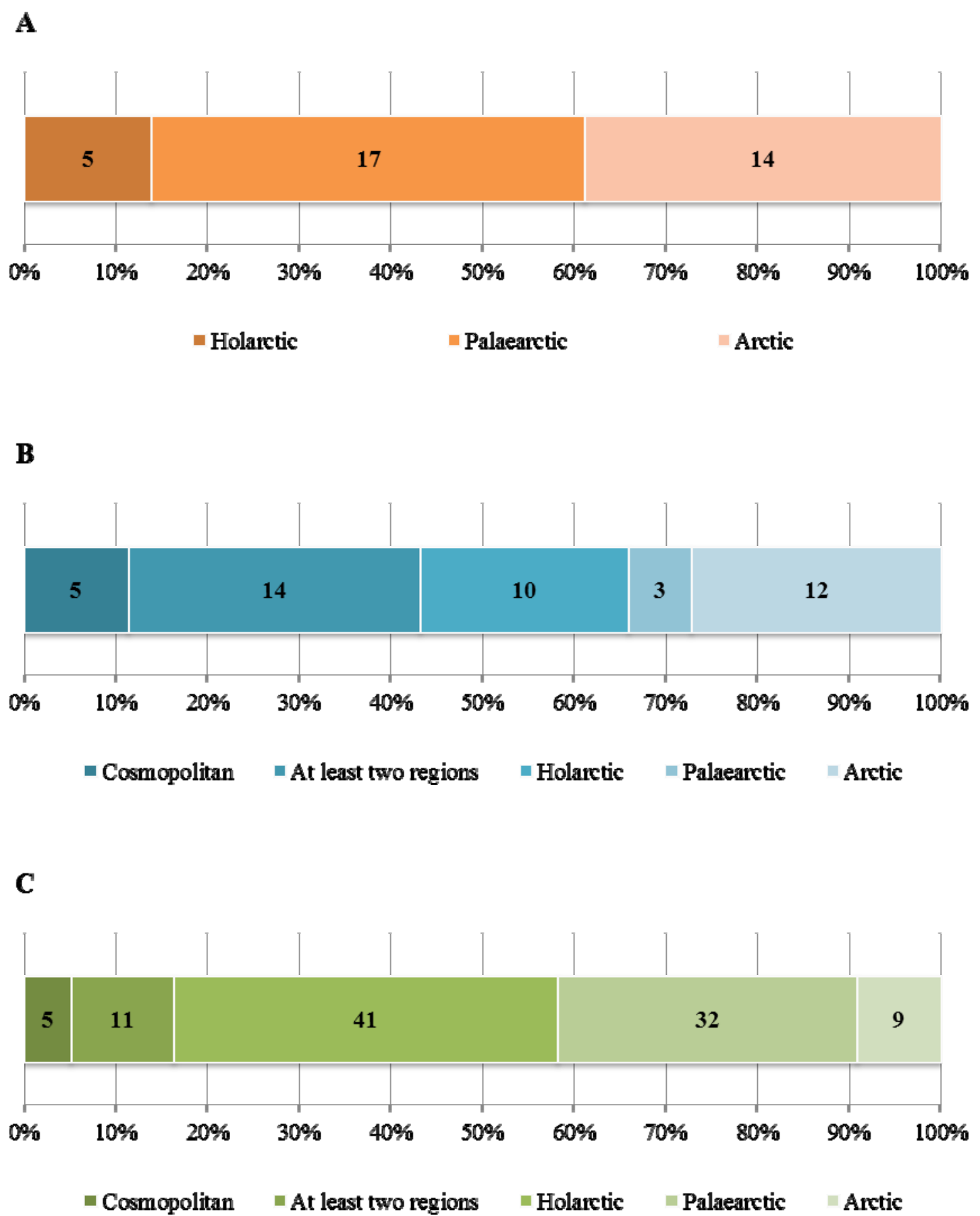

Figure 3. Distribution of mite species occurring in Svalbard: (A) Mesostigmata; (B) Trombidiformes; (C) Sarcoptiformes. No figure was made for the Ixodida, which are represented in Svalbard by one species with a distribution in "at least two regions" (see Table 1 for details).

\subsection{Trombidiformes}

The order Trombidiformes is represented in Svalbard only by the suborder Prostigmata with 17 families and 43 species recorded. The most diverse are the marine Halacaridae (12 spp.), followed by the terrestrial Bdellidae (7 spp.); these two families together comprise $44 \%$ of all the Trombidiformes known from Svalbard (Figure 1).

A strikingly large number of new species (19) have been described from Svalbard; i.e., nearly half of all Trombidiformes known from this archipelago: Bdella semiscutata Thor, 1930; Neoprotereunetes borneri (Thor, 1934); Rhagidia gelida Thorell, 1872; Bradyagaue alberti (Trouessart, 1902); Copidognathus 
poucheti (Trouessart, 1893); C. reticulatus (Trouessart, 1893); C. richardi (Trouessart, 1902); Halacarus borealis Trouessart, 1893; Thalassarachna coeca (Trouessart, 1902); T. princeps (Trouessart, 1902); T. langei Thor, 1934; T. svalbardensis Thor, 1932; Podothrombium curtipalpe (Thor, 1900); P. svalbardense Oudemans, 1930; Chenophila nanseni Skoracki \& Zawierucha, 2016; Eustigmaeus oudemansi (Thor, 1930); E. pulchellus (Thor, 1930); Bryobia borealis Oudemans, 1930; and Kerdabania arctica (Thor, 1934). Moreover, 85\% of the new species described from Svalbard have been recorded only once and mostly from early studies of the mite fauna (Table 2).

Species with wide geographical distributions predominate; cosmopolitan and Holarctic species, and those present in at least two zoogeographic regions (except that the Holarctic is treated as one unit) together comprise $66 \%$ of all the Trombidformes (Figure 2). Arctic species are also abundant representing $27 \%$ of the recorded species, while the fewest number of species have Palaearctic distributions. There are seven species which have only been recorded from Svalbard (Table 1), but these are mostly single old records of Sig Thor, including Tydeus langei, T. svalbardensis, Podothrombium svalbardense, Eustigmaeus oudemansi, E. pulchellus, and Kerdabania arctica. The one exception is the recently described Chenophila nanseni.

Trombidiformes have been found on five islands/island groups, predominantly on Spitsbergen (40 species), with others collected from Bjørnøya (11), Prins Karls Forland (6), Hopen (4), and a single species on the small islands of the Hinlopenstretet. A large number of species (27) have been found exclusively on Spitsbergen, one species [Sperchon brevirostris (Koenike, 1895)] only on Bjørnøya and two others (Thalassarachna coeca and T. princeps) only on Hopen (Table 1).

\subsection{Sarcoptiformes}

In Svalbard, 98 species of Sarcoptiformes from two suborders (Endeostigmata with 5 species and Oribatida with 93 species) have been recorded (Table 1). They represent 33 families; the richest in species is the Brachychthoniidae (14 spp.), followed by the Crotoniidae (12 spp.), Ceratozetidae (10 spp.), Oppiidae (9 spp.), and Punctoribatidae (5 spp.). Together, these five families comprise $51 \%$ of the species diversity of Sarcoptiformes in Svalbard (Figure 1). Some families are represented by only 1-4 species, yet this constitutes a significant share of their known global diversity. For example, $22 \%$ of the species belonging to the endeostigmatid family Alicorhagiidae occur in Svalbard. The oribatid families Eniochthoniidae, Peloppiidae and Tectocepheidae are also represented by large proportions of their total known species diversity, with $17 \%, 27 \%$, and $24 \%$, respectively.

Sixteen new species of Sarcoptiformes have been described from Svalbard: Alicorhagia clavipilus (Thor, 1931); A. plumipilis (Thor, 1931); Cecidophyes siedleckii Kiedrowicz, Szydlo \& Skoracka, 2016; Liochthonius sellnicki (Thor, 1930); Camisia borealis (Thorell, 1871); Hermannia reticulata Thörell, 1871; Kunstidamaeus arcticus Miko \& Monson, 2013; Ceratoppia hoeli Thor, 1930; Autogneta kaisilai Karppinen, 1967; Ameronothrus lineatus (Thorell, 1871); Oribatella arctica Thor, 1930; Ceratozetes spitsbergensis Thor, 1934; Diapterobates notatus (Thorell, 1871); Fuscozetes coulsoni A. \& S. Seniczak, 2020; Svalbardia paludicola Thor, 1930; and Chamobates birulai (Kulczynski, 1902).

Species with wide geographic ranges together form 58\% (cosmopolitan, 5\%; Holarctic, $42 \%$; and those found in at least two regions, $11 \%$ ) of the sarcoptiform species (Figure 2). Species with a Palaearctic distribution also make up a substantial proportion (33\%), with the remaining species having an Arctic distribution. Two of these Arctic species have only been found in the north of Spitsbergen: Autogneta kaisilai in Biskayerhuken and Scutozetes clavatosensillus Ermilov, Martens \& Tolstikov, 2013, in Mosselbukta; and Fuscozetes coulsoni exclusively on Edgeøya (Table 1, Figure 3). Three species have been recorded only from Svalbard and these are all recently described new species (Table 1).

Sarcoptiformes have been recorded from ten islands in the Svalbard archipelago, but not on Nordaustlandet. The most commonly collected species is Diapterobates notatus (Table 1) found on seven islands and at nearly all collecting localities. Five species [Camisia foveolata Hammer, 1955; Hermannia reticulata, H. scabra (L. Koch, 1879); Ceratoppia sphaerica (L. Koch, 1879); and Ameronothrus lineatus (Thorell, 1871)] have been found on four islands and another seven other species [Camisia horrida 
(Hermann, 1804); Mycobates sarekensis (Trägårdh, 1910); Ceratozetes spitsbergensis; Oppiella translamellata (Willmann, 1923); Tectocepheus velatus (Michael, 1880); Oribatula exilis (Nicolet, 1855); and O. tibialis (Nicolet, 1855)] on three islands. All of these species also have a wider distribution on Spitsbergen (Table 1, Figure 3).

Some species have been found exclusively on one island (Table 1), including 57 found only on Spitsbergen, four [Liochthonius clavatus (Forsslund, 1942); L. strenzkei Forsslund, 1963; Neoliochthonius piluliferus (Forsslund, 1942); and Fuscozetes coulsoni] on Edgeøya, and two [Subbelba montana (Kulczynski, 1902) and Ameronothrus nigrofemoratus (L. Koch, 1879)] from Bjørnøya.

Approximately one third of the sarcoptiform species have been recorded in Svalbard only once (Table 2). Seven of them were found in the first half of the last century and, 12 others, in the second half of the last century, including the descriptions of four new species.

\section{Discussion}

The acarofauna of the Svalbard archipelago is diverse with 178 recorded species comprising one Ixodida, 36 Mesostigmata, 43 Trombidiformes, and 98 Sarcoptiformes. However, compared with other regions of the Arctic (Alaska, Greenland, Iceland, and Taimyr), the species diversity is lower [151]. This paucity is likely to be explained by a combination of the small area of Svalbard $(60 \%$ covered by permanent snow or glaciers) [4], the greater geographic isolation, and in some cases, the more northerly locality and harsher climate. As an example, from Iceland there are 428 species of mites known, with eight Ixodida, 131 Mesostigmata, 101 Trombidiformes, and 188 Sarcoptiformes [151-153]. Although Iceland has an area only 1.5 times greater than Svalbard, the island lies at a lower latitude (between $63^{\circ}$ and $66^{\circ}$ north latitude), glaciers cover only $11 \%$ of its area, and it lies in the path of the North Atlantic Current, which results in a more ameliorated climate. The diversity of mites in Svalbard is also much poorer in comparison to mainland Norway: Ixodida comprise $8 \%$ here with Mesostigmata-15\%, Trombidiformes-13\%, and Sarcoptiformes-30\% [154-157]. However, these differences are not unexpected considering that the Svalbard archipelago is situated $900 \mathrm{~km}$ from the northern border of mainland Norway and has an area of only one fifth of that of mainland Norway [158].

The geographical isolation of islands, as a rule, results in reduced biodiversity [159], but, on the other hand, nurtures unique endemic species [160]. Svalbard has a unique flora and fauna, including endemic invertebrates: three rotifers, four tardigrades, and two aphids [86]. Fifteen mite species have been found exclusively in Svalbard, six of them being recent observations. The remaining are single historic records (i.e., from the first half of the last century) and it is consequently uncertain if any of these are endemic to Svalbard or the result of taxonomic confusion. Finding and studying the types or neotypes in Svalbard, along with more extensive sampling in the Arctic and including molecular studies, could help resolve this question.

Nevertheless, the Svalbard acarofauna is unique due to its specific Arctic elements. Amongst the Svalbard Mesostigmata in particular there are many species with an Arctic distribution (which make up nearly $40 \%$ of this group) and are similar to that observed on Greenland [151]. By contrast, the Sarcoptiformes in Svalbard are predominated by species with wide geographical ranges extending beyond the Arctic, as also seen with the Sarcoptiformes species known from Greenland [151] and northern Russia [161]. This difference between the two orders may be explained by the younger geological age of the Mesostigmata and their faster evolution rate [162]. Similarly, the Trombidiformes, which are also an ancient group [163], are dominated in Svalbard by species with wide geographic distributions. Nonetheless, Arctic species seem to be abundant among the Trombidiformes of Svalbard, comprising $27 \%$ of the species recorded, but these data should be treated with caution because they are mostly based on historic records that need to be confirmed.

Another characteristic feature of the Svalbard acarofauna is the large number of species new to science described from this region-they form approximately $25 \%$ of all mite species known from Svalbard. Seven of these species belong to the Mesostigmata, 19 to Trombidiformes, and 16 to 
Sarcoptiformes. However, many of these new species were found only once and early in the study of the mite fauna of Svalbard. These species include one mesostigmatid species, 18 Trombidiformes species (i.e., $90 \%$ of all new species from this group), and two Sarcoptiformes species. Confirming these identifications is complicated by outmoded descriptions, incomplete, or too general drawings, and by the unavailability of most type material. These species require special attention in future studies. They are possibly rare or have been wrongly identified but the possibility cannot be excluded that they have disappeared from Svalbard due to environmental changes or that they were introduced and their populations have not established in Svalbard.

Out of 36 mite species found in Svalbard only once by early workers, the majority of species (24) were collected by Sig Thor, including one new species of Mesostigmata, eight new species of Trombidiformes, and two new species of Sarcoptiformes (Table 2). Despite the widespread belief that the collection of Thor along with all his valuable type material was destroyed in accordance with Thor's wishes (see, e.g., remark 2 on page 1308 in [148]), this collection exists and is kept at the Natural History Museum in Oslo, Norway [164]. However, it is in a very poor condition and it is not certain whether the Svalbard material is in a suitable condition for re-examination.

Fortunately, some of Thor's species have been found by later sampling campaigns and redescribed, for instance, Antennoseius (Vitzthumia) oudemansi, Neoseiulus magnanalis, Proctolaelaps parvanalis, and the specimens obtained deposited in zoological collections as neotypes [28,79]. In addition, six new species of mites have been found recently in Svalbard, including two Mesostigmata-Neoseiulus grumantensis and Halolaelaps coulsoni [28,93], one Trombidiformes-Chenophila nanseni [57], and three Sarcoptiformes-Cecidophyes siedleckii, Kunstidamaeus arcticus, and Fuscozetes coulsoni $[9,67,72]$.

Recently, the first species of Ixodida, the seabird tick (Ixodes uriae), was discovered in Svalbard [76-78]. This tick is a major parasite of seabirds breeding at high latitudes and has the most extensive geographical distribution of all tick species [23], being also common throughout mainland Norway [165]. Its increasing occurence in Svalbard may be related to warming winters (the tick overwinters at the breeding sites of the seabirds) [78] as was similarly observed in Iceland with another tick species, I. ricinus Linnaeus, 1758, and which has become more common in recent years [153].

Studies conducted during the past 20 years have also added 16 species of Mesostigmata $[8,18,20,27$, $28,30,85,92,93,95,98,166]$ and 36 species of Sarcoptiformes to the Svalbard fauna $[8-10,67,71,72,74,146,167]$. This indicates that despite the relatively long history of mite studies for a region in the Arctic, our knowledge remains surprisingly poor. It is striking, however, that the Trombidiformes have been much less studied in Svalbard than other mite groups (Table 1). The reference list presented here includes only 18 papers referring to the Trombidiformes (vs. 72 papers on Sarcoptiformes and 34 on Mesostigmata), most of which were published at the end of the 19th and first half of the 20th centuries. Only three come from the present century. Therefore, it is clear that in future studies this group in particular should receive more attention.

There are also some species in Svalbard that have extraordinary disjunct distribution patterns: they occupy mainly the Arctic and parts of the subarctic regions, but are also found in some distant localities, in harsh conditions. One example is an oribatid species, Ceratozetes spitsbergensis, which has been assumed to be an Arctic species, present in Svalbard, Alaska [148], northern Canada [168,169] and the Nordic Arctic of the Russian Far East [148,170], but was found also in the Altai Mountains in Mongolia, at an altitude of 2800-3200 m a.s.l. [171] and in the Alps in Austria, at an altitude of $3300 \mathrm{~m}$ a.s.l. [172]. Since it was not found at lower elevations and/or lower latitudes, its presence in the Alps was explained by one of these theories: relict distribution [a cold-adapted species that was widely distributed in Europe during the Last Glacial Maximum (LGM), but when conditions became warmer it retreated to very restricted areas at high altitudes], or the nunatak hypothesis (survival of species on ice-free refuges) [172]. Some studies suggest that parts of Svalbard, e.g., Amsterdamøya (north-west of Svalbard), remained ice-free during the LGM [173]. It is thus possible that some invertebrates survived the LGM in situ in these glacial refugia, although, due to the harsh conditions prevailing over an 
extended period of time, it seems likely that most biota could probably not survive on nunataks [86]. Another oribatid mite with an interesting distribution is Scutozetes clavatosensillus, which has been found only in Svalbard and in Nepal (Dhaulagiri massif, 3200-3600 m a.s.l.); its distribution may also be related to glacial history. Similarly to $S$. clavatosensillus, the mesostigmatic species, Paragamasus (Aclerogamasus) insertus, may also be a glacial relict since it is known only from the Gory Stolowe Mtns., Poland $[174,175]$, where it occurred in rock crevices in very extreme conditions with long-lasting snow cover, and recently collected in Svalbard [18].

The composition of the present acarofauna of Svalbard likely results mostly from postglacial immigration [86]. The mites, being wingless, have rather low dispersal abilities. However, phoresy with migrating birds [13-17] or insects [18,95], and transport on driftwood or even direct dispersal in or on seawater with ocean currents [11], are possible dispersal pathways from the mainland to, or between, remote islands such as the Svalbard archipelago. Humans may also play a role in the dispersal of mites; as with the import of fertile agricultural soils transported from southern Russia and contemporary Ukraine to the Russian settlements on Spitsbergen (Barentsburg and Pyramiden) to enrich the soils of the greenhouses and grass lawns $[18,19]$. Mite communities in such transformed microhabitats differ noticeably from those of the adjacent Arctic tundra. Moreover, manure from abandoned cow sheds provided specific ameliorated environmental conditions enabling the survival of terrestrial invertebrate species not yet recorded elsewhere in Svalbard [18-20]. Introduction of new species to islands can be deleterious since the island communities may be disrupted, often resulting in the extinction of their endemic species [176-178].

Reconstructing the colonization of Svalbard after the LGM may be attempted by observing primary succession before retreating glaciers. For example, at the Midtre Lovén glacier foreland, two oribatid species, Camisia foveolata and Tectocepheus velatus, were the first colonizers. Mesostigmata appeared later, Proctolaelaps parvanalis being the first species recorded at this glacier foreland [10]. All these species are widely distributed in different parts of the archipelago (see Table 1). Tectocepheus velatus was also the earliest colonizer on a glacier foreland in southern Norway [179], while Camisia foveolata was one of the first colonizers on geothermally active lava fields in Iceland [180]. Oribatida are mainly saprophagous but species that are fungivores, bacteriovores, algivores, or omnivores, such as T. velatus, can find the appropriate food on seemingly baren ground [181]. In turn, the development of the first animal communities provides prey to the predatory Mesostigmata.

As shown in the present review, acarological studies of Svalbard are heavily geographically biased since most sampling has been carried out on the largest island-Spitsbergen; $90 \%$ of papers refer to this island and $90 \%$ of species have been found there (see Table 1). Almost $20 \%$ of papers refer to Bjørnøya and $25 \%$ of the species total for the archipelago have been found there, while $7 \%$ of papers refer to Edgeøya and $17 \%$ of the species are known from there. Nine other islands and island groups have been studied to a much lesser extent, with single records from other locations. It needs to be emphasized that some mites have been found exclusively on one island, other than Spitsbergen: five species on Edgeøya (Saprosecans baloghi, Liochthonius clavatus, L. strenzkei, Neoliochthonius piluliferus, and Fuscozetes coulsoni), four on Bjørnøya (Zerconopsis muestairi, Sperchon brevirostris, Subbelba montana, and Ameronothrus nigrofemoratus), and two on Hopen (Thalassarachna coeca and T. princeps), including three species new to science. It is unclear why these species have not been collected from Spitsbergen despite the more comprehensive sampling efforts on this island. The importance of further studies in different parts of the Svalbard archipelago is highlighted, in particular since the archipelago is extremely diverse geographically and climatically.

In natural conditions, the species composition and abundance of mites depends mainly on the vegetation [89], which, in turn, depends largely on the climate. For example, a relatively high mesostigmatid diversity is present along the western coast of Spitsbergen (about 30 species) [86], which experiences a comparatively mild climate for the latitude, in contrast to the polar deserts, where only five species were recorded [30]. Within the same climatic conditions, the densities of the mites also vary greatly according to the vegetation types $[22,30,88,89,136]$. 
Since mites are connected in different ways with other parts of the ecosystem, it would be particularly interesting to apply a multi-disciplinary approach to trace the effect of climate change in Svalbard. For example, one of the understudied mite groups is the family Eriophyidae. This family includes phytophagous species of great economic importance and with high invasive potential, and could be very useful for ecological studies on the effects of a changing climate at Svalbard [67]. Another poorly known group is the hyperorder Astigmata, parasitizing birds [74]. It is well-documented that climatic changes are affecting the diversity of seabirds by changing their foraging and breeding ecology, as well as increasing the abundance of temperate species [6], which could in turn affect bird-associated Astigmata.

Attention should also be paid to the least known mite order in Svalbard, the Trombidiformes, which is extremely diverse with respect to their feeding preferences (this taxon includes algivores, bacterivores, fungivores, predators, and parasites), and occupies terrestrial, freshwater, and marine habitats [182], but has not been studied at all from an ecological perspective in Svalbard.

Acarological research in the Arctic has developed rapidly in the last 20 years, markedly contributing to the knowledge of the mite communities and increasing somewhat our understanding of the factors determining these communities. Nonetheless, little is understood about the physical and morphological adaptations of mites, their adaptations to the extreme Arctic environment, or the genetic biodiversity of these isolated populations. Moreover, research has focused on the larger and more easily accessible regions of the archipelago to the detriment of the more environmentally extreme eastern and northern regions. There has also been a focus on the Sarcoptiformes and Mesostigmata while the Trombidiformes have been neglected. These areas will remain the subject of our research in the forthcoming years.

Author Contributions: Conceptualization, A.S. and D.J.G.; Investigation: Mesostigmata-D.J.G.; TrombidiformesM.D.S.; Sarcoptiformes-A.S., S.S.; Svalbard peculiarities-S.J.C.; Methodology, A.S., S.S., M.D.S., S.J.C. and D.J.G.; Visualization, A.S.; Writing — original draft, review \& editing, A.S., S.S., M.D.S., S.J.C. and D.J.G. All authors have read and agreed to the published version of the manuscript.

Funding: This research was funded by Norwegian Taxonomy Initiative: Knr 6-20.

Acknowledgments: We are grateful to Jacek Dabert (Institute of Environmental Biology, Adam Mickiewicz University, Poznań, Poland) for information about distribution of species of Astigmata, and to Reinhard Gerecke (Institute for Evolution and Ecology, University of Tübingen, Tübingen, Germany) for information about Thor's collection. This study was partly supported by the grant from The Norwegian Taxonomy Initiative - Knr 6-20, Mites of Atlantic Raised Bogs in Norway (MARB).

Conflicts of Interest: The authors declare no conflict of interest.

\section{References}

1. Gillespie, M.A.K.; Alfredsson, M.; Barrio, I.C.; Bowden, J.; Convey, P.; Coulson, S.J.; Culler, L.E.; Dahl, M.T.; Daly, K.M.; Koponen, S.; et al. Circumpolar terrestrial arthropod monitoring: A review of ongoing activities, opportunities and challenges, with a focus on spiders. Ambio 2020, 49, 704-717. [CrossRef] [PubMed]

2. Coulson, S.J. The terrestrial invertebrate fauna of the Svalbard archipelago in a changing world: History of research and challenges. Can. Entomol. 2013, 145, 131-146. [CrossRef]

3. IPCC. Summary for Policymakers. In Global Warming of $1.5^{\circ} \mathrm{C}$. An IPCC Special Report on the Impacts of Global Warming of $1.5^{\circ} \mathrm{C}$ above Pre-Industrial Levels and Related Global Greenhouse Gas Emission Pathways, in the Context of Strengthening the Global Response to the Threat of Climate Change, Sustainable Development, and Efforts to Eradicate Poverty; Masson-Delmotte, V., Zhai, P., Pörtner, H.-O., Roberts, D., Skea, J., Shukla, P.R., Pirani, A., Moufouma-Okia, W., Péan, C., Pidcock, R., et al., Eds.; Intergovernmental Panel on Climate Change: Geneva, Switzerland, 2018; pp. 3-27.

4. Hanssen-Bauer, I.; Førland, E.J.; Hisdal, H.; Mayer, S.; Sandø, A.B.; Sorteberg, A. (Eds.) Climate in Svalbard 2100-A Knowledge Base for Climate Adaptation; The Norwegian Centre for Climate Services Report 1/2019; The Norwegian Centre for Climate Services: Oslo, Norway, 2019. Available online: https://www.miljodirektoratet. no/publikasjoner/2019 (accessed on 19 April 2020). 
5. Norwegian Meteorological Institute. Precipitation in Svalbard, Hopen and Jan Mayen, Annual Total. Environmental Monitoring of Svalbard and Jan Mayen (MOSJ). 2020. Available online: http://www.mosj.no/ en/climate/atmosphere/temperature-precipitation.html (accessed on 8 August 2020).

6. Descamps, S.; Aars, J.; Fuglei, E.; Kovacs, K.M.; Lydersen, C.; Pavlova, O.; Pedersen, Å.Ø.; Ravolainen, V.; Strøm, H. Climate change impacts on wildlife in a High Arctic. Glob. Chang. Biol. 2017, 23, 490-502. [CrossRef] [PubMed]

7. Summerhayes, V.S.; Elton, C.S. Further contribution to the ecology of Spitsbergen. J. Ecol. 1928, 16, $193-268$. [CrossRef]

8. Ávila-Jiménez, M.L.; Solhøy, T.; Gwiazdowicz, D.J.; Fjellberg, A.; Dozsa-Farkas, K.; Monson, F.; De Smet, W.H.; Stur, E.; Ekrem, T.; Coulson, S.J. The terrestrial invertebrate fauna of Edgeøya, Svalbard: Arctic landscape community composition reflects biogeography patterns. Polar Biol. 2019, 42, 837-850. [CrossRef]

9. Seniczak, A.; Seniczak, S. Morphological ontogeny of Fuscozetes coulsoni sp. nov. (Acari: Oribatida: Ceratozetidae) from Svalbard, Norway. Syst. Appl. Acarol. 2020, 25, 680-696. [CrossRef]

10. Gwiazdowicz, D.J.; Zawieja, B.; Olejniczak, I.; Skubała, P.; Gdula, A.K.; Coulson, S.J. Changing microarthropod communities in front of a receding glacier in the High Arctic. Insects 2020, 11, 226. [CrossRef]

11. Coulson, S.J.; Hodkinson, I.D.; Block, W.; Webb, N.R.; Harrison, J.A. Survival of terrestrial soil-dwelling arthropods on and in seawater: Implications for trans-oceanic dispersal. Funct. Ecol. 2002, 16, 353-356. [CrossRef]

12. Coulson, S.J.; Moe, B.; Monson, F.; Gabrielsen, G.W. The invertebrate fauna of High Arctic seabird nests: The microarthropod community inhabiting nests on Spitsbergen, Svalbard. Polar Biol. 2009, 32, 1041-1046. [CrossRef]

13. Lebedeva, N.V.; Krivolutsky, D.A. Birds spread soil microarthropods to Arctic Islands. Dokl. Biol. Sci. 2003, 391, 329-332. [CrossRef]

14. Lebedeva, N.V.; Lebedev, V.D.; Melekhina, E.N. New data on the oribatid mite (Oribatei) fauna of Svalbard. Dokl. Biol. Sci. 2006, 407, 182-186. [CrossRef] [PubMed]

15. Lebedeva, N.V.; Melekhina, E.N.; Gwiazdowicz, D.J. New data on soil mites in the nests of Larus hyperboreus in the Spitsbergen archipelago. Vestn. Juzn. Naucn. Cent. Ran 2012, 8, 70-75.

16. Lebedeva, N.V.; Lebedev, V.D. Transport of oribatid mites to the polar areas by birds. In Integrative Acarology; Bertrand, M., Kreiter, S., McCoy, K.D., Migeon, A., Navajas, M., Tixier, M.S., Vial, L., Eds.; European Association of Acarologists: Montpellier, France, 2008; pp. 359-367.

17. Coulson, S.J. Association of the soil mite Diapterobates notatus (Thorell, 1871) (Acari, Oribatidae) with Cynomya mortuorum (Linnaeus, 1761) (Calliphoridae, Calliphorinae): Implications for the dispersal of oribatid mites. Int. J. Acarol. 2009, 35, 175-177. [CrossRef]

18. Coulson, S.J.; Fjellberg, A.; Gwiazdowicz, D.J.; Lebedeva, N.V.; Melekhina, E.N.; Solhøy, T.; Erséus, C.; Maraldo, K.; Miko, L.; Schatz, H.; et al. Introduction of invertebrates into the High Arctic via imported soils: The case of Barentsburg in the Svalbard. Biol. Invasions 2013, 15, 1-5. [CrossRef]

19. Coulson, S.J.; Fjellberg, A.; Gwiazdowicz, D.J.; Lebedeva, N.V.; Melekhina, E.N.; Solhøy, T.; Erséus, C.; Maraldo, K.; Miko, L.; Schatz, H.; et al. The invertebrate fauna of anthropogenic soils in the High-Arctic settlement of Barentsburg; Svalbard. Polar Res. 2013, 32, 19273. [CrossRef]

20. Coulson, S.J.; Fjellberg, A.; Melekhina, E.N.; Taskaeva, A.A.; Lebedeva, N.V.; Belkina, O.; Seniczak, S.; Seniczak, A.; Gwiazdowicz, D.J. Microarthropod communities of industrially disturbed or imported soils in the High Arctic; the abandoned coal mining town of Pyramiden, Svalbard. Biodivers. Conserv. 2015, 24, 1671-1690. [CrossRef]

21. Hughes, K.A.; Convey, P. The protection of Antarctic terrestrial ecosystems from inter and intra-continental transfer of non-indigenous species by human activities: A review of current systems and practices. Glob. Environ. Chang. 2010, 20, 96-112. [CrossRef]

22. Seniczak, S.; Seniczak, A.; Gwiazdowicz, D.J.; Coulson, S.J. Community structure of oribatid and gamasid mites (Acari) in moss-grass tundra in Svalbard (Spitsbergen, Norway). Arct. Antarct. Alp. Res. 2014, 46, 591-599. [CrossRef]

23. Muñoz-Leal, S.; González-Acuña, D. The tick Ixodes uriae (Acari: Ixodidae): Hosts, geographical distribution, and vector roles. Ticks tick-borne Dis. 2015, 6, 843-868. [CrossRef]

24. Krantz, G.W.; Walter, D.E. A Manual of Acarology, 3rd ed.; Texas Tech University Press: Lubbock, TX, USA, 2009; pp. 1-807. 
25. Beaulieu, F.; Dowling, A.P.G.; Klompen, H.; de Moraes, G.J.; Walter, D.E. Superorder Parasitiformes Reuter, 1909. In Animal Biodiversity: An Outline of Higher-Level Classification and Survey of Taxonomic Richness; Zhang, Z.-Q., Ed.; Magnolia Press: Auckland, New Zealand, 2011; pp. 123-128. [CrossRef]

26. Fauna Europaea. Available online: https://fauna-eu.org (accessed on 19 April 2020).

27. Gwiazdowicz, D.J.; Teodorowicz, E.; Coulson, S.J. Redescription of Zercon solenites Haarløv, 1942 (Acari, Zerconidae) with a key to the Svalbard species of the genus Zercon. Int. J. Acarol. 2011, 37 (Suppl. 1), 135-148. [CrossRef]

28. Kolodochka, L.A.; Gwiazdowicz, D.J. A new species of predaceous mite of the genus Neoseiulus Hughes (Acari, Phytoseiidae), with redescriptions of N. magnanalis (Thor) and N. ellesmerei (Chant \& Hansell), from Svalbard, High Arctic. Zootaxa 2014, 3793, 441-452.

29. Gwiazdowicz, D.J.; Rakowski, R. Redescription of Proctolaelaps parvanalis (Thor, 1930) (Acari: Ascidae) from Spitsbergen. Entomol. Fenn. 2009, 20, 281-286. [CrossRef]

30. Ávila-Jiménez, M.L.; Gwiazdowicz, D.J.; Coulson, S.J. The mesostigmatid mite (Acari: Parasitiformes) fauna of Svalbard: A revised inventory of a high Arctic Archipelago. Zootaxa 2011, 3091, 33-41. [CrossRef]

31. Zhang, Z.-Q.; Fan, Q.-H.; Pesic, V.; Smit, H.; Bochkov, A.V.; Khaustov, A.A.; Baker, A.; Wohltmann, A.; Wen, T.H.; Amrine, J.W.; et al. Order Trombidiformes Reuter, 1909. In Animal Biodiversity: An Outline of Higher-Level Classification and Survey of Taxonomic Richness; Zhang, Z.-Q., Ed.; Magnolia Press: Auckland, New Zealand, 2011; pp. 129-138. [CrossRef]

32. Bolton, S.J.; Chetverikov, P.E.; Klompen, H. Morphological support for a clade comprising two vermiform mite lineages: Eriophyoidea (Acariformes) and Nematalycidae (Acariformes). Syst. Appl. Acarol. 2017, 22, 1096-1131. [CrossRef]

33. Klimov, P.B.; OConnor, B.M.; Chetverikov, P.E.; Bolton, S.J.; Pepato, A.R.; Mortazavi, A.L.; Tolstikov, A.V.; Bauchan, G.R.; Ochoa, R. Comprehensive phylogeny of acariform mites (Acariformes) provides insights on the origin of the four-legged mites (Eriophyoidea), a long branch. Mol. Phylogenet. Evol. 2018, 119, 105-117. [CrossRef] [PubMed]

34. Hernandes, F.A.; Skvarla, M.J.; Fisher, J.R.; Dowling, A.P.G.; Ochoa, R.; Ueckermann, E.A.; Bauchan, G.R. Catalogue of snout mites (Acariformes: Bdellidae) of the world. Zootaxa 2016, 4152, 1-83. [CrossRef] [PubMed]

35. Skvarla, M.J.; Fisher, J.R.; Dowling, A.P. A review of Cunaxidae (Acariformes, Trombidiformes): Histories and diagnoses of subfamilies and genera, keys to world species, and some new locality records. ZooKeys 2014, 418, 1-103. [CrossRef] [PubMed]

36. Khaustov, A.A. A new genus and species in the mite family Eupodidae (Acari, Eupodoidea) from Crimea. ZooKeys 2014, 422, 11-22. [CrossRef] [PubMed]

37. Szudarek-Trepto, N.; Kazmierski, A.; Dabert, M.; Dabert, J. Molecular phylogeny of Eupodidae reveals that the family Cocceupodidae (Actinotrichida; Eupodoidea) and its genus Filieupodes are valid taxa. Exp. Appl. Acarol. 2020, 80, 43-57. [CrossRef] [PubMed]

38. Meyer, M.K.P.; Ryke, P.A.J. Mites of the superfamily Eupodoidea (Acarina: Prostigmata) associated with South African plants. S. Afr. J. Agric. Sci. 1960, 3, 481-496.

39. Strandtmann, R.W.; Davies, L. Eupodiform mites from Possession Island, Crozet Islands, with a key to the species of Eupodes (Acarina: Prostigmata). Pac. Insects 1972, 14, 39-56.

40. Umina, P.A.; Hoffman, A.A.; Weeks, A.R. Biology, ecology and control of the Penthaleus species complex (Acari: Penthaleidae). Exp. Appl. Acarol. 2004, 34, 211-237. [CrossRef] [PubMed]

41. Jesionowska, K. A morphological study of the genus Penthalodes (Acari, Prostigmata, Eupodoida, Penthalodidae) with description of a new species. Zootaxa 2010, 2672, 29-49. [CrossRef]

42. Zacharda, M. Soil mites of the family Rhagidiidae (Actinedida: Eupodoidea). Morphology, sytematics, ecology. Acta Univ. Carol. Biol. 1980, 516, 489-785.

43. Zacharda, M.; Gude, M.; Kraus, S.; Hauck, C.; Molenda, R.; Růžička, V. The relict mite Rhagidia gelida (Acari, Rhagidiidae) as a biological cryoindicator of periglacial microclimate in European Highland Screes. Arct. Antarct. Alp. Res. 2005, 37, 402-408. [CrossRef]

44. Qin, T.K. A checklist and key to species of Eupodoidea (Acari: Prostigmata) from Australia and New Zealand and their subantarctic islands. J. R. Soc. N. Z. 1998, 28, 295-307. [CrossRef]

45. Bartsch, I. Checklist of marine and freshwater halacarid mite genera and species (Halacaridae: Acari) with notes on synonyms, habitats, distribution. Zootaxa 2009, 1998, 1-170. [CrossRef] 
46. André, H.M. Redefinition of the genus Triophtydeus Thor, 1932 (Acari: Actinedida). Zool. Meded. 1985, 59, 189-195.

47. Thor, S. Acarina-Tydeidae, Ereynetidae; Das Tierreich, Walter de Gruyter: Berlin, Germany, 1933; Volume 60, pp. 1-84.

48. Makarova, O.; Behan-Pelletier, V. 18.3.6. Oribatida (=Cryptostigmata, Beetle Mites). In The Greenland Entomofauna; Böcher, J., Kristensen, N.P., Pape, T., Vilhelmsen, L., Eds.; An Identification Manual of Insects, Spiders and Their Allies; Koninklijke Brill NV: Leiden, The Netherlands, 2015; pp. 802-845.

49. André, H.M. In search of the true Tydeus (Acari, Tydeidae). J. Nat. Hist. 2005, 39, 975-1001. [CrossRef]

50. Silva, G.L.; Metzelthin, M.H.; Silva, O.S.; Ferla, N.J. Catalogue of the mite family Tydeidae (Acari: Prostigmata) with the world key to the species. Zootaxa 2016, 4135, 1-68. [CrossRef]

51. Baker, E.W. A review of the genera of the family Tydeidae (Acarina). Adv. Acarol. 1965, 2, 95-133.

52. André, H.M. A generic revision of the family Tydeidae (Acari: Actinedida). IV. Generic descriptions, keys and conclusions. Bull. Ann. Soc. Belg. Ent. 1980, 116, 103-130.

53. Makarova, O.L. Acarocenoses (Acariformes, Parasitiformes) in polar deserts: 1. Mite assemblages of the Severnaya Zemlya Archipelago: Structure of fauna and abundance. Entomol. Rev. 2002, 82, 839-856.

54. Abe, H. Annotated checklist of Japanese water mites (Acari: Prostigmata: Hydracarina). Acta Arachnol. 2005, 54, 111-145. [CrossRef]

55. Esen, Y.; Pesic, V.; Erman, O. Water mites of the genus Sperchon Kramer (Acari: Hydrachnidia: Sperchontidae) from Turkey, with description of two new species. Zootaxa 2010, 2514, 35-46. [CrossRef]

56. Mąkol, J.; Wohltmann, A. An annotated checklist of terrestrial Parasitengona (Actinotrichida: Prostigmata) of the world, excluding Trombiculidae and Walchiidae. Ann. Zool. 2012, 62, 359-562. [CrossRef]

57. Skoracki, M.; Zawierucha, K. Chenophila nanseni sp. n. (Acari: Syringophilidae) parasitising the barnacle goose in Svalbard. Pol. Polar Res. 2016, 37, 121-130. [CrossRef]

58. Beron, P. Acarorum Catalogus VII. Trombidiformes: Prostigmata: Raphignathoidea. Fam. Barbutiidae, Caligonellidae, Camerobiidae, Cryptognathidae, Dasythyreidae, Dytiscacaridae, Eupalopsellidae, Homocaligidae, Mecognathidae, Raphignathidae, Stigmaeidae, Xenocaligonellididae. Pensoft Natl. Mus. Nat. Hist. Sofia Bulg. 2020. [CrossRef]

59. Pritchard, A.E.; Baker, E.W. A Revision of the Spider Mite Family Tetranychidae; Memoirs Series; Pacific Coast Entomological Society: San Francisco, CA, USA, 1955; Volume 2.

60. Migeon, A.; Dorkeld, F. Spider Mites Web: A Comprehensive Database for the Tetranychidae. 2020. Available online: http://www1.montpellier.inra.fr/CBGP/spmweb (accessed on 2 July 2020).

61. Khaustov, A.A. A description of new genus, Kerdabania gen. n. with four new species (Acari: Heterostigmata: Neopygmephoridae). Acarina 2009, 17, 171-188.

62. Schatz, H.; Behan-Pelletier, V.M.; O'Connor, B.M.; Norton, R.A. Suborder Oribatida van der Hammen, 1968. In Animal Biodiversity: An Outline of Higher-Level Classification and Survey of Taxonomic Richness; Zhang, Z.-Q., Ed.; Magnolia Press: Auckland, New Zealand, 2011; pp. 141-148.

63. Beaulieu, F.; Knee, W.; Nowell, V.; Schwarzfeld, M.; Lindo, Z.; Behan-Pelletier, V.M.; Lumley, L.; Young, M.R.; Smith, I.; Proctor, H.C.; et al. Acari of Canada. In The Biota of Canada-A Biodiversity Assessment. Part 1: The Terrestrial Arthropods; Langor, D.W., Sheffield, C.S., Eds.; ZooKeys: Sofia, Bulgaria, 2019; Volume 819, pp. 77-168. [CrossRef]

64. Walter, D.E.; Bolton, S.; Uusitalo, M.; Zhang, Z.-Q. Suborder Endeostigmata Reuter, 1909. In Animal Biodiversity: An Outline of Higher-Level Classification and Survey of Taxonomic Richness; Zhang, Z.-Q., Ed.; Magnolia Press: Auckland, New Zealand, 2011; pp. 139-140.

65. Uusitalo, M. Terrestrial species of the genus Nanorchestes (Endeostigmata: Nanorchestidae) in Europe. In Trends in Acarology; Sabelis, M.W., Bruin, J., Eds.; Springer: Dordrecht, The Netherlands, 2010; pp. 161-166. [CrossRef]

66. Thor, S.; Willmann, C. Acarina. 7la Eupodidae, Penthalodidae, Penthaleidae, Rhagidiidae, Pachygnathidae, Cunaxidae. Das Tierreich 1941, 71, 1-186.

67. Kiedrowicz, A.; Rector, B.G.; Zawierucha, K.; Szydło, W.; Skoracka, A. Phytophagous mites (Acari: Eriophyoidea) recorded from Svalbard, including the description of a new species. Polar Biol. 2016, 39, 1359-1368. [CrossRef] 
68. Subías, L.S. Listado Sistemático, Sinonímico y Biogeográfico de los Ácaros Oribátidos (Acariformes, Oribatida) del Mundo (excepto fósiles) (15 a actualisación). Graellsia 2004, 2020, 60, (número extraordinario), 3-305 (2004), actualized pdf in 2020. Available online: http://bba.bioucm.es/cont/docs/RO_1.pdf (accessed on 14 April 2020).

69. Seniczak, S.; Seniczak, A. Differentiation of external morphology of Oribatulidae (Acari: Oribatida) in light of the ontogeny of three species. Zootaxa 2012, 3184, 1-34. [CrossRef]

70. Seniczak, A.; Seniczak, S. Morphological ontogeny of Achipteria punctata (Acari: Oribatida: Achipteriidae). Zootaxa 2018, 4540, 54-72. [CrossRef]

71. Bayartogtokh, B.; Schatz, H.; Ekrem, T. Distribution and diversity of the soil mites of Svalbard, with redescriptions of three known species (Acari: Oribatida). Int. J. Acarol. 2011, 37, 467-484. [CrossRef]

72. Miko, L.; Monson, F.D. Two interesting damaeid mites (Acari, Oribatida, Damaeidae Berlese, 1896) from the British Isles and Svalbard (Spitsbergen, Norway), with a description of Kunstidamaeus arcticus n.sp. Acarologia 2013, 53, 89-100. [CrossRef]

73. Klimov, P.B. A review of acarid mites of the tribe Caloglyphini (Acaridae, Acariformes) with description of a new genus and species from Siberia and Russian Far East. Vestn. Zool. 2000, 34, 27-35.

74. Dabert, M.; Coulson, S.J.; Gwiazdowicz, D.J.; Moe, B.; Hanssen, S.A.; Biersma, E.; Pilskog, H.E.; Dabert, J. Differences in speciation progress in feather mites (Analgoidea) inhabiting the same host: The case of Zachvatkinia and Alloptes living on arctic and long-tailed skuas. Exp. Appl. Acarol. 2015, 65, 163-179. [CrossRef]

75. Dabert, J. Personal Communication; Adam Mickiewicz University: Poznan, Poland, 2020.

76. McCoy, K.D. Consequences of Dispersal in Host-Parasite Systems: Population Dynamics, Genetic Structure, and Localadaptation of the Seabird tickixodes Uriae. Ph.D. Thesis, Pierreand Marie Curie University, Paris, France, 2001.

77. Coulson, S.J.; Lorentzen, E.; Strøm, H.; Gabrielsen, G.W. The parasitic tick Ixodes uriae (Acari: Ixodidae) on seabirds from Spitsbergen, Svalbard. Polar Res. 2009, 28, 399-402. [CrossRef]

78. Descamps, S. Winter temperature affects the prevalence of ticks in an Arctic sea-bird. PLoS ONE 2013, 8, e65374. [CrossRef]

79. Teodorowicz, E.; Gwiazdowicz, D.J.; Coulson, S.J. Redescription of Antennoseius (Vitzthumia) oudemansi (Acari, Mesostigmata) from Spitsbergen, Svalbard. Entomol. Fenn. 2014, 25, 27-42. [CrossRef]

80. Gwiazdowicz, D.J.; Teodorowicz, E.; Coulson, S.J. Redescription of Arctoseius haarlovi Lindquist, 1963 (Acari: Ascidae) from Spitsbergen. Entomol. Fenn. 2011, 22, 140-148. [CrossRef]

81. Trouessart, E. Note sur les Acariens recueillis au Spitzberg pendant le voyage de la Manche. Nouv. Arch. Miss. Sci. Litt. 1893, 5, 255-263.

82. Trouessart, E. Révision des Acariens des regions Arctiques et description d'espèces nouvelles. M. Soc. Natl. Sci. Nat. Math. Cherb. 1895, 29, 183-206.

83. Zmudczyńska-Skarbek, K.; Barcikowski, M.; Drobniak, S.M.; Gwiazdowicz, D.J.; Richard, P.; Skubała, P.; Stempniewicz, L. Transfer of ornithogenic influence through different trophic levels of the Arctic terrestrial ecosystem of Bjørnøya (Bear Island), Svalbard. Soil Biol. Biochem. 2017, 115, 475-489. [CrossRef]

84. Makarova, O.L. Gamasid mites (Parasitiformes, Mesostigmata) of the European Arctic and their distribution patterns. Entomol. Rev. 2013, 93, 113-133. [CrossRef]

85. Gwiazdowicz, D.J.; Gulvik, M.E. Mesostigmatid mites (Acari, Mesostigmata) in Svalbard. In Materiały XXXII Międzynarodowego Sympozjum Polarnego; Uniwersytet Wrocławski, Wydział Nauk o Ziemi i Kształtowania Środowiska: Wrocław, Poland, 2008; pp. 32-34.

86. Coulson, S.J.; Schatz, H.; Gwiazdowicz, D.J.; Solhøy, T. On the oribatid and mesostigmatid mites (Acari) of the High Arctic island of Hopen. Pol. Polar Res. 2014, 35, 133-139. [CrossRef]

87. Coulson, S.J.; Fjellberg, A.; Snazell, R.; Gwiazdowicz, D.J.; Ávila-Jiménez, M.L. On the Collembola, Araneae and Gamasida from the Kinnvika region of Nordaustlandet, Svalbard. Geogr. Ann. A 2011, 93, 253-257. [CrossRef]

88. Byzova, J.B.; Uvarov, A.V.; Petrova, A.D. Seasonal changes in communities of soil invertebrates in tundra ecosystems of Hornsund, Spitsbergen. Pol. Polar Res. 1995, 16, 245-266.

89. Gwiazdowicz, D.J.; Coulson, S.J. High-Arctic gamasid mites (Acari, Mesostigmata): Community composition on Spitsbergen, Svalbard. Polar Res. 2011, 30, 8311. [CrossRef] 
90. Pilskog, H.E.; Solhøy, T.; Gwiazdowicz, D.J.; Grytnes, J.A.; Coulson, S.J. Invertebrate communities inhabiting nests of migrating passerine, wild fowl and sea birds breeding in the High Arctic, Svalbard. Polar Biol. 2014, 37, 981-998. [CrossRef]

91. Thor, S. Beiträge zur Kenntnis der Invertebratenfauna von Svalbard. Skr. Svalbard Ishavet 1930, 27, 1-156.

92. Gwiazdowicz, D.J.; Solhøy, T.; Coulson, S.J.; Lebedeva, N.V.; Melekhina, E.N. First record of Vulgarogamasus immanis (Acari, Mesostigmata) in Svalbard. Pol. Polar Res. 2012, 33, 35-39. [CrossRef]

93. Gwiazdowicz, D.J.; Teodorowicz, E. Description of Halolaelaps coulsoni n. sp. (Acari, Halolaelapidae) from the High Arctic. Acarologia 2017, 57, 393-406. [CrossRef]

94. Hirschmann, W. Die Gattung Halolaelaps Berlese \& Trouessart 1889 nov. comb. Acarologia 1966, 9, 21-24.

95. Gwiazdowicz, D.J.; Coulson, S.J. First record of Thinoseius spinosus (Acari, Eviphididae) from the High Arctic Island of Spitsbergen (Svalbard) including a key to deutonymphs of genus Thinoseius. Int. J. Acarol. 2010, 36, 233-236. [CrossRef]

96. Makarova, O.L. To the study of mites of the genus Arctoseius Thor (Parasitiformes, Ascidae) from the Far North: 3. Ranges and ecological preferences of species. Entomol. Rev. 2000, 80, 143-150.

97. Lindquist, L.L.; Makarova, O.L. Two new circumpolar mite species of the genus Arctoseius (Parasitiformes, Mesostigmata, Ascidae). Entomol. Rev. 2011, 91, 1054-1072. [CrossRef]

98. Gwiazdowicz, D.J.; Coulson, S.J.; Grytnes, J.A.; Pilskog, H.E. The bird ectoparasite Dermanyssus hirundinis (Acari, Mesostigmata) in the High Arctic; a new parasitic mite to Spitsbergen, Svalbard. Acta Parasitol. 2012, 57, 378-384. [CrossRef]

99. Summerhayes, V.S.; Elton, C.S. Contributions to the ecology of Spitsbergen and Bear Island. J. Ecol. 1923, 11, 214-286. [CrossRef]

100. Krumpál, M.; Cyprich, D.; Zejda, J.; Ambros, M. The occurrence of field vole (Microtus arvalis Pallas 1778) and its acarofauna on Spitsbergen (Svalbard). Biologia 1991, 46, 881-885.

101. Hull, M.A. On some land mites (Acari) from Spitzbergen and Bjørnøya. Results of the Oxford University Expedition to Spitsbergen, 1921. Ann. Mag. Nat. Hist. 1922, 10, 621-623. [CrossRef]

102. Thorell, T. Om Arachnider från Spetsbergen och Beeren-Eiland. Öfvers. Kongl. Vetensk.-Akad. Förh. 1871, 6, 683-702.

103. Thor, S. Neue Beiträge zur Kenntnis der Invertebraten-Fauna vin Svalbard. Zool. Anz. 1934, 107, 114-139.

104. Bertram, G.C.L.; Lack, D. Notes on the animal ecology of Bear Island. J. Anim. Ecol. 1938, 7, 27-52. [CrossRef]

105. Trägårdh, I. Beiträge zur Fauna der Bären-Insel. Bihang till K. Svenska Vet. Akad. Handlingar. 1900, 26, 1-24.

106. Haarløv, N. A morphologic-systematic-ecological investigation of Acarina and other representatives of the microfauna of the soil around Mørkefjord, Northeast Greenland. Medd. Grønl. 1942, 128, 1-71.

107. Trouessart, E. Note préliminaire sur les acariensmarins (Halacaridae) recueillis par S.A.S. le Prince de Monaco, dans les mersarctiques. Bull. Soc. Zool. Fr. 1902, 27, 66-70.

108. Barr, W. The Helgoland Expedition to Svalbard: Die Deutsche Expedition in das Nordliche Eismeer, 1898. Arctic 1988, 41, 203-214. [CrossRef]

109. Chertoprud, E.S.; Makarova, O.L.; Novichkova, A.A. First data on aquatic mites (Acari) of inland water bodies of West Spitsbergen, Svalbard. Acarina 2017, 25, 181-189. [CrossRef]

110. Thor, S. Norwegische Tydeidae VIII-XV, bemerkungen uber die Gattung Tydeus und uber Augen, Tracheen usw. Zool. Anz. 1932, 98, 69-91.

111. Soar, C.D. A species of Hydracarina found at Bear Island, June 17th, 1921-The Oxford University Expedition to Spitsbergen, 1921. Microscopy 1922, 14, 301-304.

112. Oudemans, A.C. Acarologische Aanteekeningen. XCIX. Entomol. Ber. 1930, 8, 11-20.

113. Thor, S. Norwegische Alycidae I-VII. Zool. Anz. 1931, 194, 229-238.

114. Niedbała, W. Oribatei (Acari) of Spitsbergen. B. Pol. Acad. Sci.-Biol. 1971, 19, 737-742.

115. Karppinen, E. Notes on the arthropod fauna of Spitsbergen. Acta Entomol. Fenn. 1967, 33, $18-26$.

116. Forsslund, K.H. Notizen über Oribatei (Acari). I. Ark. Zool. 1957, 2, 583-593.

117. Forsslund, K.H. Liochthonius muscorum n. sp. und L. lapponicus (Träg.) (Acari, Oribatei). Entomol. Tidskr. 1964, 85, 236-239.

118. Byzova, J.B.; Uvarov, A.V. Mites of the tundra biotopes in the vicinity of bird colonies on Spitsbergen. In The 6th all-Union Meeting on the Problems of Theoretical and Applied Acarology. Aszchabad, Turkmenistan. Abstracts; Balashov, Y.S., Ed.; Russian Academy of Science: Saint Petersburg, Russia, 1990; pp. 152-153. 
119. Coulson, S.J. Terrestrial and freshwater invertebrate fauna of the high Arctic Archipelago of Svalbard. Zootaxa 2007, 1448, 41-58. [CrossRef]

120. Coulson, S. Personal Communication; Swedish University of Agricultural Sciences: Uppsala, Sweden, 2020.

121. Coulson, S.J.; Hodkinson, I.D.; Strathdee, A.T.; Bale, J.S.; Block, W.; Worland, M.R.; Webb, N.R. Simulated climate change: The interaction between vegetation type and microhabitat temperatures at Ny Ålesund, Svalbard. Polar Biol. 1993, 13, 67-70. [CrossRef]

122. Coulson, S.J.; Hodkinson, I.D.; Block, W.; Webb, N.R.; Worland, M.R. Low summer temperatures: A potential mortality factor for high arctic soil microarthropods? J. Insect Physiol. 1995, 41, 783-792. [CrossRef]

123. Coulson, S.J.; Hodkinson, I.D.; Webb, N.R.; Block, W.; Bale, J.S.; Strathdee, A.T.; Worland, M.R.; Wooley, C. Effects of experimental temperature elevation on high-arctic soil microarthropod populations. Polar Biol. 1996, 16, 147-153. [CrossRef]

124. Coulson, S.J.; Leinaas, H.P.; Ims, R.A.; Søvik, G. Experimental manipulation of the winter surface ice layer-The effects on a High Arctic soil microarthropod community. Ecography 2000, 23, 299-306. [CrossRef]

125. Coulson, S.J.; Hodkinson, I.D.; Webb, N.R. Microscale distribution patterns in high Arctic soil microarthropod communities: The influence of plant species within the vegetation mosaic. Ecography 2003, 26, 801-809. [CrossRef]

126. Hodkinson, I.D.; Coulson, S.J.; Webb, N.R.; Block, W.; Strathdee, A.T.; Bale, J.S. Feeding studies on Onychiurus arcticus (Tullberg) (Collembola: Onychiuridae) on West Spitsbergen. Polar Biol. 1993, 14, 17-19. [CrossRef]

127. Webb, N.R.; Coulson, S.J.; Hodkinson, I.D.; Block, W.; Bale, J.S.; Strathdee, A.T. The effects of experimental temperature elevation on populations of cryptostigmatic mites in high Arctic soils. Pedobiologia 1998, 42, 298-308.

128. Hodkinson, I.D.; Bird, J.M. Anoxia tolerance in high Arctic terrestrial microarthropods. Ecol. Entomol. 2004, 29, 506-509. [CrossRef]

129. Dollery, R.; Hodkinson, I.D.; Jónsdóttir, I.S. Impact of warming and timing of snow melt on soil microarthropod assemblages associated with Dryas-dominated plant communities in Svalbard. Ecography 2006, 29, 111-119. [CrossRef]

130. Kulczyński, V. Zoologische Ergebnisse der russischen Expeditionen nach Spitzbergen. Annu. Mus. Zool. Acad. Imp. Sci. St. Petersb. 1902, 7, 347-354.

131. Solhøy, I.W. A redescription of Mycobates sarekensis (Trägårdh) (Acari: Oribatei). Acarologia 1997, 38, 69-77.

132. Block, W. Some arctic Oribatei. Acarologia 1966, 8, 161-162.

133. Trägårdh, I. Monographie der arktischen Acariden. Fauna Arct. 1904, 4, 1-78.

134. Seniczak, S.; Seniczak, A.; Coulson, S.J. Morphological ontogeny, distribution and descriptive population parameters of Hermannia reticulata (Acari: Oribatida: Hermanniidae), with comments on Crotonioidea. Int. J. Acarol. 2017, 43, 52-72. [CrossRef]

135. Seniczak, S.; Seniczak, A.; Coulson, S.J. Morphological ontogeny and distribution of Hermannia scabra (Acari: Oribatida: Hermanniidae) in Svalbard and descriptive population parameters. Acarologia 2017, 57, 877-892. [CrossRef]

136. Seniczak, S.; Plichta, W. Structural dependence of moss mite populations on patchiness of vegetation in moss-lichen-tundra at the north coast of Hornsund, West Spitsbergen. Pedobiologia 1978, 18, 145-152.

137. Gordeeva, E.V. Moritzoppiella gen. n.-A new genus of oribatid mites of the family Oppiidae (Oribatei, Acariformes). Vestn. Zool. 2000, 34, 17-26.

138. Hayward, S.A.L.; Worland, M.R.; Bale, J.S.; Convey, P. Temperature and the hygropreference of the Arctic collembolan Onychiurus arcticus and mite Lauroppia translamellata. Physiol. Entomol. 2000, 25, 266-272. [CrossRef]

139. Thor, S. Übersicht der norwegischen Cryptostigmata mit einzelnen Neben bemerkungen. NYT Mag. Nat. 1937, 77, 275-307.

140. Schubart, H. Morphologische Grundlagen für die Klärung der Verwandtschaftsbeziehungen innerhalb der Milbenfamilie Ameronothridae (Acari, Oribatei). Zoologica 1975, 123, 23-91.

141. Søvik, G.; Leinaas, H.P. Variation in extraction efficiency between juvenile and adult oribatid mites: Ameronothrus lineatus (Oribatida, Acari) in a Macfadyen high-gradient canister extractor. Pedobiologia 2002, 46, 34-41. [CrossRef]

142. Søvik, G.; Leinaas, H.P. Adult survival and reproduction in an arctic mite, Ameronothrus lineatus (Acari, Oribatida): Effects of temperature and winter cold. Can. J. Zool. 2003, 81, 1579-1588. [CrossRef] 
143. Søvik, G.; Leinaas, H.P. Long life cycle and high adult survival in an arctic population of the mite Ameronothrus lineatus (Acari, Oribatida) from Svalbard. Polar Biol. 2003, 26, 500-508. [CrossRef]

144. Søvik, G. Observations on ovoviviparity and mixed-parity mode in arctic populations of Ameronothrus lineatus (Acari, Oribatida). Acarologia 2003, 43, 393-398.

145. Søvik, G.; Leinaas, H.P.; Ims, R.A.; Solhøy, T. Population dynamics and life history of the oribatid mite Ameronothrus lineatus (Acari, Oribatida) on the high Arctic Archipelago of Svalbard. Pedobiologia 2003, 47, 257-271. [CrossRef]

146. Seniczak, S.; Seniczak, A.; Graczyk, R.; Tømmervik, H.; Coulson, S.J. Distribution and population characteristics of the soil mites Diapterobates notatus and Svalbardia paludicola (Acari: Oribatida: Ceratozetidae) in High Arctic Svalbard (Norway). Polar Biol. 2017, 40, 1545-1555. [CrossRef]

147. Seniczak, S.; Seniczak, A.; Coulson, S.J. Morphology, distribution and certain population parameters of the Arctic mite Oribatella arctica (Acari: Oribatida: Oribatellidae). Int. J. Acarol. 2015, 41, 395-414. [CrossRef]

148. Behan-Pelletier, V.M. Ceratozetidae of the western North American Arctic. Can. Entomol. 1985, 117, 1287-1366. [CrossRef]

149. Seniczak, S.; Seniczak, A.; Coulson, S.J. Morphology, distribution and biology of Mycobates sarekensis (Acari: Oribatida: Punctoribatidae). Int. J. Acarol. 2015, 41, 663-675. [CrossRef]

150. Fain, A.; Camerik, A.M. Notes on the mites of the genus Ereynetes Berlese (Acari: Ereynetinae), with description of five new species from South Africa. Bull. Inst. R. Sci. Nat. Belg. Entomol. 1994, 64, 145-164.

151. Makarova, O.L. The fauna of free-living mites (Acari) of Greenland. Entomol. Rev. 2015, 95, 108-125. [CrossRef]

152. Gjelstrup, P.; Solhøy, T. Oribatid mites (Acari). In The Zoology of Iceland, Steenstrupia 3; Zoological Museum University of Copenhagen: Copenhagen, Denmark, 1994; Volume 57e, pp. 1-78.

153. Alfredsson, M.; Olafsson, E.; Eydal, M.; Unnsteinsdottir, E.R.; Hansford, K.; Wint, W.; Alexander, N.; Medlock, J.M. Surveillance of Ixodes ricinus ticks (Acari: Ixodidae) in Iceland. Parasit. Vectors 2017, 10, 466. [CrossRef]

154. Mehl, R. Checklist of Norwegian ticks and mites (Acari). Fauna Norv. Ser. B 1979, 26, 31-45.

155. Gwiazdowicz, D.J.; Gulvik, M.E. Checklist of Norwegian mesostigmatid mites (Acari, Mesostigmata). Nor. J. Entomol. 2005, 52, 117-125.

156. Seniczak, A.; Bolger, T.; Roth, S.; Seniczak, S.; Djursvoll, P.; Jordal, B.H. Diverse mite communities (Acari: Oribatida, Mesostigmata) from a broadleaf forest in western Norway. Ann. Zool. Fenn. 2019, 56, 121-136. [CrossRef]

157. Seniczak, A.; Seniczak, S.; Iturrondobeitia, J.C.; Solhøy, T.; Flatberg, K.I. Diverse Sphagnum mosses support rich moss mite communities (Acari, Oribatida) in mires of western Norway. Wetlands 2019. [CrossRef]

158. The Editors of Encyclopaedia Britannica, Svalbard. Encyclopædia Britannica, Inc. Available online: https://www.britannica.com/place/Svalbard (accessed on 19 April 2020).

159. Veron, S.; Haevermans, T.; Govaerts, R.; Mouchet, M.; Pellens, R. Distribution and relative age of endemism across islands worldwide. Sci. Rep. 2019, 9, 11693. [CrossRef] [PubMed]

160. Losos, J.B.; Ricklefs, R.E. Adaptation and diversification on islands. Nature 2009, 457, 830-836. [CrossRef] [PubMed]

161. Melekhina, E.N. Taxonomic diversity and areology of oribatid mites (Oribatei) of the European North of Russia. Proc. Komi Sci. Cent. Ural BranchRas 2011, 2, 30-37.

162. Makarova, O.L.; Böcher, J. Diversity and geographical ranges of Greenland mites (Acari: Oribatida and Mesostigmata). In Species and Communities in Extreme Environments; Pensoft Publisher and KMK Science Press: Sofia, Moscow, Russia, 2009; pp. 165-186.

163. Dabert, M.; Witaliński, W.; Kaźmierski, A.; Olszanowski, Z.; Dabert, J. Molecular phylogeny of acariform mites (Acari, Arachnida): Strong conflict between phylogenetic signal and long-branch attraction artifacts. Mol. Phylogenet. Evol. 2010, 56, 222-241. [CrossRef]

164. Gerecke, R. Personal Communication; University of Tübingen: Tübingen, Germany, 2019.

165. Mehl, R.; Traavik, T. The tick Ixodes uriae (Acari, Ixodides) in seabird colonies in Norway. Fauna Norv. Ser. B 1983, 30, 94-107.

166. Gwiazdowicz, D.J.; Coulson, S.J.; Ávila-Jiménez, M.L. First records of Zercon andrei Sellnick, 1958 and Zerconopsis muestairi Schweizer, 1949) (Acari, Mesostigmata) from Bjørnøya, Svalbard. Nor. J. Entomol. 2009, 56, 117-119. 
167. Seniczak, A.; Seniczak, S. Diversity of oribatid mites (Sarcoptiformes) in the Svalbard Archipelago: A historical overview. Zootaxa 2020, 4834, 41-65. [CrossRef]

168. Behan-Pelletier, V.M. Oribatid mites (Acari: Oribatida) of the Yukon. In Insects of the Yukon. Biological survey of Canada (Terrestrial Arthropods); Danks, H.V., Downes, J.A., Eds.; Canadian Museum of Nature: Ottawa, Canada, 1997; pp. 115-149.

169. Behan-Pelletier, V.; Schatz, H. Patterns of diversity in the Ceratozetoidea (Acari: Oribatida): A North American assessment. In Trends in Acarology; Sabelis, M.W., Bruin, J., Eds.; Springer: Dordrecht, The Netherlands, 2010; pp. 97-104. [CrossRef]

170. Ryabinin, N.A. Oribatid mites (Acari, Oribatida) in soils of the Russian Far East. Zootaxa 2015, 3914, $201-244$. [CrossRef] [PubMed]

171. Bayartogtokh, B. Fauna and communities of oribatid mites of Mongolia (Acari: Oribatida). Ph.D. Thesis, Russian Academy of Sciences, Moscow, Russia, 2007.

172. Fischer, B.M.; Schatz, H.; Querner, P.; Pauli, H. Ceratozetes spitsbergensis Thor, 1934: An Arctic mite new to continental Europe (Acari: Oribatida). Int. J. Acarol. 2016, 42, 135-139. [CrossRef]

173. Landvik, J.Y.; Brook, E.J.; Gualtieri, L.; Raisbeck, G.; Salvigsen, O.; Yiou, F. Northwest Svalbard during the last glaciation: Ice-free areas existed. Geology 2003, 31, 905-908. [CrossRef]

174. Micherdziński, W. Die Familie Parasitidae Oudemans 1901 (Acarina, Mesostigmata); Państwowe Wydawnictwo Naukowe: Kraków, Poland, 1969; pp. 1-690.

175. Kamczyc, J.; Gwiazdowicz, D.J. Soil mites (Acari, Mesostigmata) from Szczeliniec Wielki in the Stołowe Mountains National Park (SW Poland). Biol. Lett. 2009, 46, 21-27. [CrossRef]

176. Frenot, Y.; Chown, S.L.; Whinam, J.; Selkirk, P.; Convey, P.; Skotnicki, M.; Bergstrom, D. Biological invasions in the Antarctic: Extent, impacts and implications. Biol. Rev. 2005, 80, 45-72. [CrossRef]

177. Bergstrom, D.M.; Lucieer, A.; Kiefer, K.; Wasley, J.; Belbin, L.; Pedersen, T.K.; Chown, S.L. Indirect effects of invasive species removal devastate World Heritage Island. J. Appl. Ecol. 2009, 46, 73-81. [CrossRef]

178. Convey, P. Terrestrial biodiversity in Antarctica-Recent advances and future challenges. Polar Sci. 2010, 4, 135-147. [CrossRef]

179. Hågvar, S.; Solhøy, T.; Mong, C.E. Primary succession of soil mites (Acari) in a Norwegian glacier foreland, with emphasis on oribatid species. Arct. Antarct. Alp. Res. 2009, 41, 219-227. [CrossRef]

180. Buda, J.; Olszanowski, Z.; Wierzgoń, M.; Zawierucha, K. Tardigrades and oribatid mites in bryophytes from geothermally active lava fields (Krafla, Iceland) and the description of Pilatobius islandicus sp. nov. (Eutardigrada). Pol. Polar Res. 2018, 39, 425-453. [CrossRef]

181. Hågvar, S.; Pedersen, A. Food choice of invertebrates during early glacier foreland succession. Arct. Antarct. Alp. Res. 2015, 47, 561-572. [CrossRef]

182. Walter, D.E.; Proctor, H.C. Mites: Ecology, Evolution \& Behaviour; Springer: Dordrecht, The Netherlands; Heidelberg, Germany; New York, NY, USA; London, UK, 2013. [CrossRef] 